\title{
La inflación en El Salvador
}

\author{
Roberto Rivera Campos*
}

Este documento presenta los resultados de una investigación empirica sobre el proceso inflacionario en El Salvador, el cual se ha extendido desde 1973 hasta la fecha. Siendo la inflación uno de los problemas críticos recientes, es natural que varios estudios se hayan realizado sobre el tema ${ }^{1}$, algunos con mayor y otros con menor evidencia empírica. Como es usual en este tipo de investigaciones económicas, las perspectivas teóricas desde las cuales se ha abordado el problema han sido variadas ${ }^{2}$. Cuesliones lales como el papel de la demanda, el financiamiento del déficit fiscal, la devaluación, los estrangulamientos económicos y otros más, han recibido distinios énfasis como determinantes de la inflación.

Estos elementos y otros más serán considerados en la presente invesligación. En el conlexto de una economia subdesarrollada, pequeña, abierla y olgopólica, nuestra hipótesis principal afirmará que en El Salvador la inflación ha sido fundamentalmente un fenómeno de costos. Esta hipólesis se argumentará en dos niveles: primeramente, sobre la base de algunos estudios previos que han tratado de evidenciar empiricamente el comportamiento real de los encargados de fijar los precios de los diferentes tipos de bienes; y enseguida, sobre la base de los resultados empíricos obtenidos de modelos econométricos alternalivos.

Una breve descripción del argumento que se desarrollará más adelante puede lacilitar ya interpretación de esta investigación. Siendo que la manufaclura salvadoref́na, predominantemente oligopólica, tiende a operar con capacidad ociosa y a compelir con importaciones sustitutas, los aumenlos de demanda no se traducen solamente en aumentos de precios sino tämbién en incremenlos de la capacidad instalada utilizada y en más importaciones. De esta manera, los precios de los bienes manulaclurados no se pueden fijar conlorme a la inleracción de la oferla y la demanda doméstica. Estos, en consecuencia, tienden a reflejar el 
comportamiento de los coslos y de los margenes de ganancia de los empresarios. Por otro lado, en la agricullura, por el contrario, debido a sus restricciones particulares ${ }_{1}$ los excesos de demanda tienden a determinar el comportamiento de los precios; sin embargo; debido a que una pequena economia abierla dispone de la poșibilidad de liquidar estos excesos por medio de importaciones, el mecanismo de fijación de precios agricola opera de manera restringida. Los precios internacionales, los precios de garantia y los costos de producción, ganan, de esta manera, influencia considerable en ta dinámica de los precios agrícolas. Los excesos de demanda ya sea que provengan del sector público o del privado, más que incidir en el alza de los precios, impactan positivamente sobre la oferta y negativamente sobre la cuenta extema. Estos serán los supuestos de comportamiento sobre los cuales se desarrollará la presente investigación.

En la próxima sección se presenta un primer escrutunio de la realidad económica, analizando los determinantes principales de los precios en los diferentes sectores productivos. Aqui se suministra alguna evidencia empírica sobre el comportamiento y dinamismo de los sectores manulaclurero, agricola, financiero, constructor y externo. En la segunda sección, sobre la base de lo argumentado en la primera, se elabora un modelo econométrico de la fijación de precios por coslos. En la segunda parte de la sección el modelo se somete a verificación empírica y se analizan los resultados. En la última sección, se pasa revista a algunos modelos economélricos monetaristas que en el pasado se aplicaron a las economias latinoamericanas y uno que se aplicó a la economia salvadoreha. Asimismo, estos modelos se veritican empíricamenle en el período actual y los resultados se contraslan con los obtenidos en la sección anterior. La investigación finaliza con algunas conclusiones.

\section{Formaclón de preclos en la economía salvadorena}

Después de 15 anos de notable estabilidad de precios entre 1958 y 1972, El Salvador entró a una etapa que se ha caracterizado por el alza ininterrumpida de los precios, la cual se extiende hasla el presente. Es este último periodo el objeto del presente estudio. El gráfico de la página siguiente muestra el proceso inflacionario reciente, donde se aprecia momentos en que los precios se han acelerado y momentos en que han permanecido creciendo a tasas relativamente uniformes.

Existen en el país varios indicadores del crecimiento de los precios. El indice de precios al por mayor que se publica en dos versiones, segun que incluya o excluya los precios del café; el indice de los precios implícilos del PIB; y el indice de precios al consumidor. Este último es un índice ponderado de los precios de una canasta básica de mercancias y servicios que en su diversidad refleja el consumo promedio de la po- 
Inflación en El Salvador

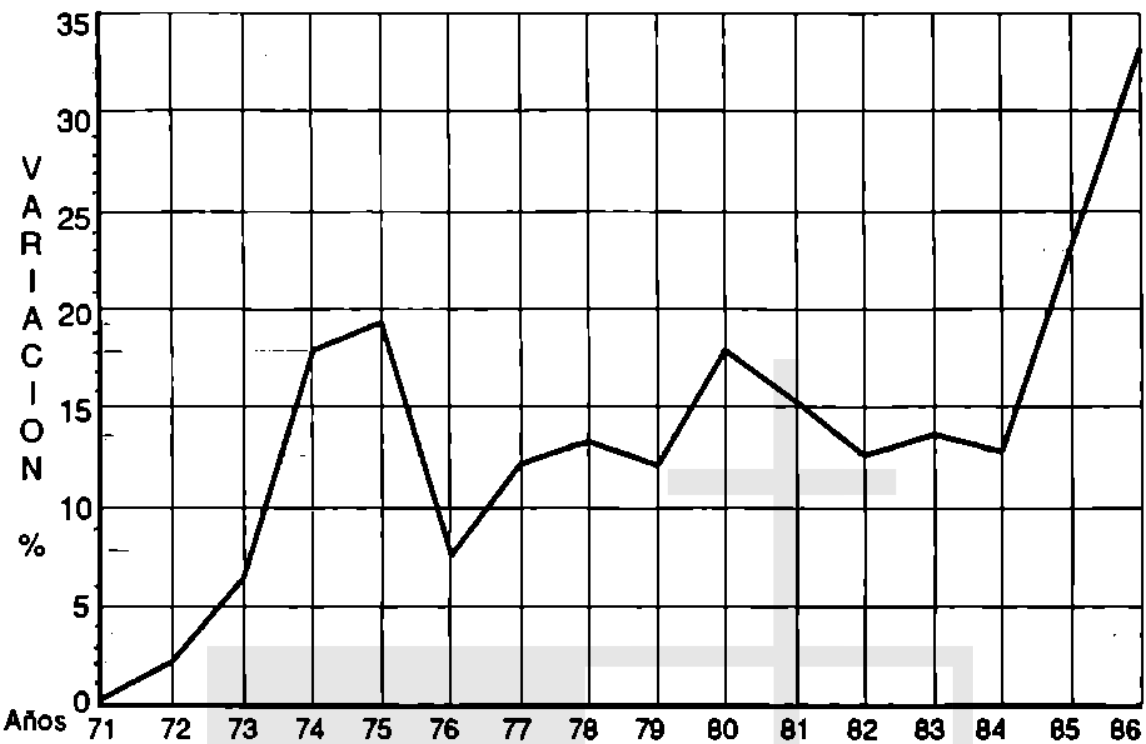

blación. Esta canasta está constituida por cuatro tipos de bienes: alimentos, vestuario, vivienda y misceláneos Un ejercicio cuantitativo permitió estimar el peso que cada uno de estos componentes tiene en el indice de precios al consumidor. El resultado fue el siguiente:

$\log I P C=0.55 \log I P A L+0.11 \log I P V E+0.16 \log |P V|+0.18 \log I P M I$

$\mathrm{R} 2=0.99$

donde IPC = indice de precios al consumidor

IPAL = indice de precios de los alimentos

IPVE = Indice de precios del vestuario

IPVI = Indice de precios de la vivienda

IPMI = indice de precios misceláneos

el cual nos muestra que los alimentos constituyen el componente principal en la canasta básica. La ecuación muestra que una variación de $1 \%$ en los precios de los alimentos hará variar el Indice de precios del consumidor en $0.55 \%$.

La forma más usual de presentar la ińlación es a través del indice de precios al consumidor; parece, en consecuencia, conveniente utilizar a lo 
largo de la presente investigación este índice. La incidencia de los precios de los diferenles tipos de bienes en los precios al consumidor nos indica que una manera razonable de iniciar el esludio de la inflación es analizando el comportamiento de los precios de estos bienes. En las siguientes subsecciones estudiaremos los determinantes principales de los precios en los sectores agricola, manutacturero y de la consirucción; asimismo estudiaremos algunas caracterlsticas de los mercados externos, de Irabajo y financiero por la incidencia que parecen haber tenido en el proceso inflacionario.

\section{La formación de preclos en la agrlcultura}

Dentro de la agricultura salvadoreña se distinguen dos sectores perfeclamente diferenciados. El primero de ellos, dedicado a la exportación (caté, algodón y caf́a de azúcar), se desarrolla bajo dirección capilalista, sobre grandes extensiones de tierra de propiedad privada y utilizando mano de obra abundante a la cual no alcanza a suministrarle empleo permanente. Los precios de estos productos de exporlación se rigen por el mercado mundial (sector tomador de precios) y están sujetos a amplias flucluaciones debido a las grandes variaciones de la oferta mundial. Su influencia, sin embargo, hacia el interior de la economia, en términos de precios, es considerablemente insigniticante, aunque indirectamente a través de los cambios que provoca en la liquidez nacional puede llegar a influir signiticalivamente. Este efecto posible to consideraremos más adelante al desarrollar el mercado tinanciero.

El segundo subsector agricola es el de producción de alimentos para el consumo doméstico. Esle es desde el punto de vista de su incidencia en los precios del consumidor el más imporlante. Predominantemente, la producción de alimenlos de origen agricola se desarrolla por aquellos que no encuentran subsislencia pemanente trabajando como mano de obra en el seclor exporlador; en consecuencia, esta producción se realiza en pequefras parcelas de tierra generalmente arrendadas y con el objeto principal de producir para la auto subsistencia, dejando para el mercado el excedente. Asimismo, el acceso de estos productores de granos básicos al crédito oficial ha estado considerablemente restringido, aunque, por supuesto, algunas instituciones crediticias oliciales existen desde hace algunos años con el objeto de facilitar dicho acceso a los pequeños campesinos. Generalmente el pequeño produclor tiene que recurrir a financiar su producción a través de los intermediarios a quienes debe pagar tasas de interés considerablemente superiores a las oliciales. Un estudio reciente (Ladman 1986) sobre los mercados financieros rurales mostraba que mientras la tasa de interés oficial ascendia al $12 \%$ anual los intermediarios oblenian $24 \%$, muchas veces sobre préstamos que ellos obtenian de los mismos bancos de crédito rural. 
La política de precios del gobierno respecto a este sector, por otra parte, ha estado orientada a mantenerlos a niveles relativamente bajos; esta política se ha llevado adelante mediante la creación del Inslituto Regulador de Abastecimienlo, el cual fija precios de garantla para el pequefio productor. La capacidad real de este Instituto para regular los precios ha sido bastanle limitada debido al poco volumen de producto que maneja, se estima que alcanza a comprar y distribuir solamente el $20 \%$ de toda la producción de granos básicos. El $80 \%$ por to tanto, se vende generalmente por arriba del precio de garantía, no por los productores directos, sino por los mismos intermediarios, quienes adquieren los productos pagando bajos precios a los productores, muchas veces por ataduras impuestas al momento de concederles el crédito, y vendiéndolos por arriba de los precios oficiales.

En estas circunslancias, la oferta agricola alimenticia doméstica ha carecido del dinamismo necesario para acompanar a la expansión de la demanda. El cuadro siguiente muestra este lenlo dinamismo entre 1971 y 1986.

$\begin{array}{lcc}\begin{array}{c}\text { Tasas de crecimiento promedio anual de la producción } \\ \text { de granos }\end{array} & \\ \text { grano } & 1971 / 1979 & 1979 / 1986 \\ & & \\ \text { maiz } & 4.1 & -0.8 \\ \text { frijol } & 2.7 & 1.0 \\ \text { arroz } & 1.4 & 1.1 \\ \text { maicillo } & 0.4 & -0.5\end{array}$

Fuente: calculado en base a datos obtenidos de la Dirección General de Economia Agropecuaria

En una economía cerrada este comportamiento tendria que dar lugar a presiones inflacionarias, debido a que la naluraleza de la producción agrícola no permite expandir la olerta sino hasla después de una temporada. Los excesos de demanda agricola, en consecuencia, se liquidan a través de los precios (Kalecki 1982). No ocurre asl en una economia abierta como la salvadorefía, con bastante acceso a los mercados internacionales de productos agrícolas y con una politica gubernamental de cubrir por medio de importaciones los délicits de producción.

En un esludio realizado a fines de la década pasada (Siri y Dominguez 1979), mostraban que entre 1960 y 1976 el gobierno salvadoreno se apoyó en los suministros extranjeros de granos básicos en un monlo 
promedio equivalente el $22 \%$ de la producción doméstica y observan que el indice de producción tiende a estar sistemáticamente correlacionadio de manera inversa con la tasa de imporlaciones del siguiente periodo, dado que el ciclo de producción termina en septiembre determinando de esta manera las disponibilidades del siguiente período. Esta afirmación sigue teniendo vigencia en la actualidad. Frenle a la reciente sequia que alectó en este ano la oferla doméstica de Irijol, el gobierno reaccionó imporlando de Costa Rica los quintales necesarios. En el siguiente cuadro se muestra de qué manera el sector externo ha estado sirviendo de estabilizador de la olerta nacional de maiz.

$\begin{array}{lrr}\text { Producción doméstica e importaciones netas de maiz } \\ \text { (miles de quintales) } \\ \text { período } & \text { producción } & \text { imporlación neta* } \\ & & \\ 1973 / 74 & 8815.6 & -55.6 \\ 1974 / 75 & 7668.5 & 505.2 \\ 1975 / 76 & 9548.8 & 10.9 \\ 1976 / 77 & 7444.1 & 185.1 \\ 1977 / 78 & 8255.2 & 1580.2 \\ 1978 / 79 & 11020.6 & 959.1 \\ 1979 / 80 & 11364.5 & 388.9 \\ 1980 / 81 & 11447.7 & 443.3 \\ 1981 / 82 & 10867.8 & 28.0 \\ 1982 / 83 & 9000.0 & 624.9 \\ 1983 / 84 & 9633.0 & 714.7 \\ 1984 / 85 & 11461.5 & 106.8 \\ 1985 / 86 & 107692 & 514.1 \\ 1986 / 87 & 9500.0 & 373.6 \\ & & \end{array}$

Fuente: Dirección General de Economla Agropecuaria

- negativo significa exportación nela

El sector externo ha funcionado, pues, como una válvula que transforma gran parte de las presiones inflacionarias de las restricciones agricolas en déficits de balanza de pagos ${ }^{3}$.

Parece plausible, por consiguienle, poslular que los faclores que más influyen en la determinación del crecimienlo de los precios agricolas son los costos de producción, los precios internacionales y los precios de garanlia oficiales, aunque eslos úllimos, a su vez, se eslablecen considerando a los dos primeros. 


\section{La formación de preclos en la manufactura}

Al igual que en la mayoria de los paises subdesarrollados, la manulactura salvadorefia presenta una estructura trunca en el sentido de que predomina la producción de bienes de consumo de fácil elaboración, a la vez que se articula pobremente con la producción agrícola. Estas características condicionan a la manufactura a una dependencia externa extrema de bienes intermedios y de capilal. Según la matriz insumo producto de 1978, el coeficiente de insumos industriales (de la rama 14 a la 34) que provenian del sector primario (de la rama 1 a la 13) era solamente 0.06 . Esto signilica que por cada unidad de valor producida en la manufaclura solo $6 \%$ provenia de la agricultura. Asimismo, el $52 \%$ de lodas las ramas manufactureras importaban en promedio el $47.2 \%$ de lodos sus insumos.

Una característica más del sector que merece deslacarse es su estruclura oligopólica Según estimaciones de Rivera C. (1983), por lo menos $31.6 \%$ del valor de la producción en 1978 se producia bajo formas oligopolicas de mercado. El siguiente cuadro presenta al segmento oligopólico de la manufactura salvadorena.

\section{Monopolios y oligopolios en la manufactura}

$\begin{array}{lcc}\text { Grupo } & \begin{array}{l}\text { Indice de } \\ \text { Herfindahl }\end{array} & \text { Forma de mercado } \\ \text { Aceites y grasas } & 0.30 & \text { oligopolio abierto } \\ \text { Productos de molineria } & 0.34 & \text { " cerrado } \\ \text { Alimentos diversos } & 0.45 & \text { abierto } \\ \text { Bebidas malteadas } & 1.00 & \text { monopolio } \\ \text { Bebidas gaseosas } & 0.34 & \text { oligopolio cerrado } \\ \text { Abonos y plaguicidas } & 0.50 & " \text { " abierto } \\ \text { Jabones, perfumes y cosmet. } & 0.35 & \text { monopolio } \\ \text { Ref. de petróleo } & 1.00 & \text { oligopolio cerrado } \\ \text { Cemento } & 0.50 & \text { oligopolio abierto } \\ \text { Prod. minerales no met. } & 0.26 & \end{array}$

Fuente: Rivera C. (1983).

Es sumamente importante destacar este grado de oligopolización de la manulactura salvadoreha en la presenle investigación, pues ha sido suficientemente evidenciado que las estrucluras oligopólicas operan con características muy especificas respecto a otras estructuras (Kalecki 1982, Steindl 1979). En primer lugar, estratégicamente tienden a operar 
con capacidad ociosa. Esto significa que las plantas son disenadas ingenierilmente con un cierto margen de capacidad adiclonal para hacer frente a las variaciones temporales e imprevislas de la demanda; por el contrario, las variaciones sostenidas de la demanda por arriba de este nivel planeado se enfrentan con nuevas inversiones para adecuar la capacidad instalada. Según un estudio reciente (Pérez y Contreras 1987), en el cual se entrevistó a los encargados de fijar los precios en 40 empresas manulactureras, distribuidas en pequenas, medianas, grandes y gigantes, el $62.5 \%$ de ellas respondió de que sl operaban con capacidad ociosa y que lo hacian asl aún en tiempos normales (no de crisis económica) para entrentar variaciones imprevistas en la demanda. Es notable que el $78 \%$ de las empresas gigantes tiende a operar con capacidad ociosa4.

Este resultado empírico sobre el coeficiente de capacidad utilizada tiene implicaciones negalivas para el pensamienlo tradicional acerca de la reacción de las empresas frente a las variaciones de la demanda 5 . Según la teoria Iradicional, los excesos de demanda de corto plazo se liquidan a través de aumentos de precios debido a que se parte del supuesto de que las empresas operan a plena capacidad, no es asi en nuestro caso, pues aquellos tienden a liquidarse de dos maneras: expandiendo la capacidad utilizada de la planta y/o aumentando la importación de bienes extranjeros competitivos. De esta manera, debe reconocerse que en una economia pequeńa, abierta y oligopóllca, la presión inflacionaria directa de los excesos de demanda de bienes industriales tiende a translormarse en presiones de aumento de la producción y de déficit de balanza de pagos.

La alimación anterior debe tomarse con cuidado, pues siempre que aparezcan rigideces para ampliar la producción o para importar, por ejemplo por carencia de insumos, de créditos, de divisas, etc., la presión inflacionaria intema será el resultado de los excesos de demanda. No parece ser este el caso normal de la economia salvadorena; en la década pasada no hubo escaces de divisas y en la aclual, el programa de apoyo a la balanza de pagos de la AID ha mantenido la capacidad importadora del pais aún en medio de la crisis. Las entrevistas nos mostraron que el $95 \%$ de las empresas de la muestra'reaccionaban aumentando la producción frente a aumentos en la demanda y sólo el 5\% respondió que incrementaba los precios.

En las condiciones anteriores los precios no pueden determinarse por la interacción de la olerta y de la demanda, sino por el costo de producción. "Los precios los lijamos agregando un margen de ganancia pre establecido a los costos de producción", respondió el $92.5 \%$ de todas las empresas entrevistadas. Solamente una empresa respondió que lo hacía igualando el costo marginal y el ingreso marginal, dos más no 
dieron respuesta concreta. Ciertamente, los precios de los bienes manufacturados se forman agregando un margen de ganancia que es considerado normal por la empresa a los costos de los insumos nacionales e importados, de los salarios y del financiamiento, es decir de los intereses que hay que pagar por los préstamos bancarios y extra bancarios para capital de trabajo, y de la depreciación del equipo (Kalecki 1982, Hall y Hitch 1939, Labini 1966, Godley y Nordhaus 1972, Taylor 1983).

Un resultado importante de las encuestas muestra que el $\mathbf{5 3 \%}$ de las empresas tienen periodos de revisión de los precios (trimestral, semes(ral o anual), razón por la cual se ven obligados en un contexto inflacionario e incierto como el nuestro a ajuslar por adelantado los precios para prolegerse de la inflación futura.

En resumen, pues, los tactores que más parecen incidir en la formación de los precios de los bienes manufacturados son: los precios de los insumos importados, el costo salarial, el costo del financiamiento y las expectalivas inflacionarias. Analicemos en seguida el comportamiento de algunos de eslos factores.

\section{IPC $\bullet$ IPC de soclos de comerclo} (variaciones relativas anuales)

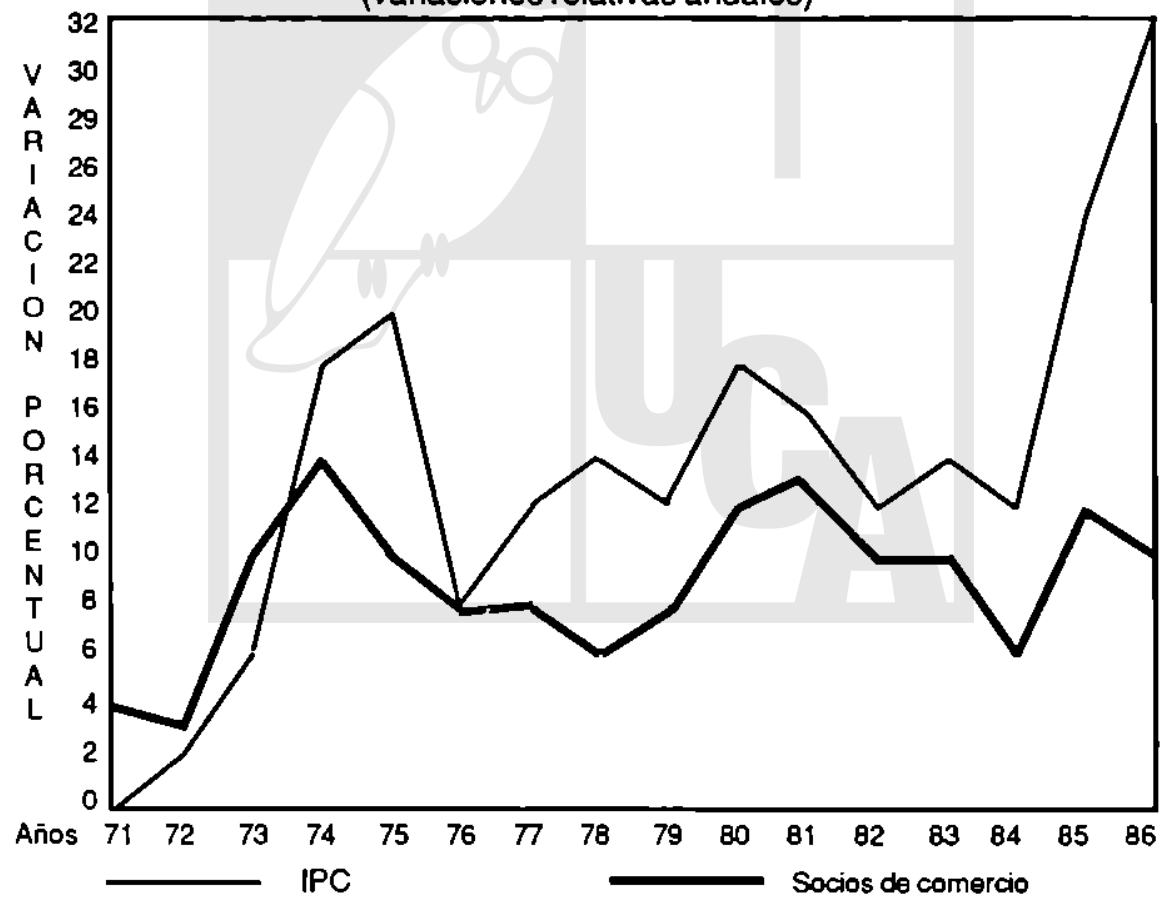




\subsection{El mercado de Insumos Internaclonal}

Ya hemos senalado la dependencia de la manufactura salvadorena de las importaciones y el efecto signiticativo de sus precios sobre los costos de producción domésticos. En este sentido hablamos de una inflación importada. El peso de la inflación mundial en el proceso inflacionario reciente se percibe claramente en el siguiente gráfico. El indicador de precios internacionales que hemos utilizado es el ponderado de los precios de los 5 principales socios comerciales.

Es altamerte significativa la asociación enlre los ciclos de ambas curvas. Las primeras dos crestas inflacionarias están asociadas a las dos crisis pelroleras; la primera, de septiembre de 1973 a finales de 1974, durante la cual se cuadriplicaron los precios del crudo; y la segunda de finales de 1979 a junio de 1980, en la cual se duplicaron. Entre estas dos crisis la presión inflacionaria provenienle' del exterior, aunque no desapareció, sí se alenuó relativamenle. A partir de 1981 la presión inflacionaria nuovamente ha descendido sostenidamente, debido al descenso de los precios del petróleo. Las expectativas del fuluro de los precios del pelróleo es que tenderán a mantenerse bajos.

\subsection{El mercado de trabajo}

Al igual que en la mayoria de los paises subdesarrollados, el mercado de trabajo en El Salvador se caracteriza por el excedente de oferta de mano de obra y por la debilidad relativa de sus organizaciones laborales, lo cual determina que los salarios se mantengan secularmente deprimidos. El Salario en consecuencia es regulado por el Estado a través de la vigencia del salario mínimo. Este se eslablece de manera diversificada, existiendo actualmente salarios mínimos para la agricultura, para la recolección y procesamiento de los produclos de exportación, para la manufaclura, para la construcción y para los servicios; éstos a su vez se encuentran diferenciados, algunas veces, según se trate de trabajo masculino o femenino y olras, como ocurre con el trabajo en la manulactura, según la localidad.

Los salarios corrientes sin embargo pueden fijarse por encima del salario mínimo correspondiente, dependiendo sobre todo de las relaciones obrero patronal; es decir, de la capacidad que tienen los sindicatos en empresas especificas de elevar sus salarios por encima del nivel minimo. A pesar de eslas divergencias reales el comportamiento del salario minimo es un buen indicador del comportamienlo del nivel general de salarios en el país, sobre lodo si estamos analizando tasas de crecimiento.

En estas circunstancias, los salarios han jugado un papel pasivo dentro del proceso inflacionario. El ajuste salarial se ha dado en res- 
puesta a la inflación misma, en tanto que ésta ha ido deteriorando progresivamente la capacidad adquisitiva de los trabajadores y con ello sus condiciones de vida. La primera revisión al salario mínimo después de muchos anos fue la de mediados de 1973, ciertamente antes de la primera alza de los precios mundiales del pelróleo, pero después de la crisis agricola de 1972 en el país y en el mundo, la cual se convirtio en presión inflacionaria desde el inicio de 1973. No obstante, los aumentos salariales, aunque en respuesta a la misma inflación, se relroalimentan en nueva fuente inflacionaria en los períodos siguientes por el aumento de costos que signilican para las empresas.

Salarios nominales y reales en la manufactura

\begin{tabular}{|c|c|c|c|c|c|c|}
\hline & 1973 & 1975 & 1977 & 1979 & 1981 & 1983 \\
\hline $\begin{array}{l}\text { salario nominal } \\
\text { salario real } \\
\text { (precio de 1975) }\end{array}$ & $\begin{array}{l}4.10 \\
5.71\end{array}$ & $\begin{array}{l}6.20 \\
6.20\end{array}$ & $\begin{array}{l}7.00 \\
5.85\end{array}$ & $\begin{array}{l}9.00 \\
5.73\end{array}$ & $\begin{array}{r}11.00 \\
5.20\end{array}$ & $\begin{array}{r}11.00 \\
4.11\end{array}$ \\
\hline
\end{tabular}

Fuente: Ministerio de Trabajo y Previsión Social, Departamen to de Planificación.

El cuadro anterior nos confirma lo dicho antes. Los trabajadores salvadorenos han ido por detrás de la inflación reaccionando frente a ella pero de manera insuliciente, no impidiendo el deterioro progresivo de su capacidad adquisitiva. Este es el papel inflacionario pasivo que le asignamos a los salarios.

\subsection{El mercado financlero}

Una característica de los paises subdesarrollados es la necesidad de linanciar a través de préstamos bancarios o extra bancarios un monto considerable de capital de trabajo. No exislen investigaciones al respecto sobre las empresas salvadorefias, sin embargo algunos estudios realizados para otros paises (Taylor 1982, van Wijnbergen 1982, Lim 1987), indican que tales montos ascienden alrededor de $10 \%$. Una de las causas explicativas de esta especificidad de nuestras economias es el poco desarrollo de los sistemas financieros. No existe, por ejemplo en EI Salvador, una bolsa de valores donde las empresas puedan colocar sus acciones para financiar sus inversiones; en el sistema bancario el crédito es limitado y poco ágil; y el acceso al crédito es difícil para muchas empresas. Esto ha dado lugar a que surjan mercados inlormales, los cua- | les generalmente salislacen las necesidades financieras ágilmente' aunque con inlereses más elevados ${ }^{6}$.

Aunque el sistema bancario aplique una política monetaria pasiva que 
regula las tasas de interés activas, la existencia de mercados financieros informales en donde ésla se fija por la olerta y la demanda de créditos, hace que la tasa de interés ponderada en ambos mercados fluclúe. Este hecho llama la atención pues permite ver que, conlrario a la visión tradicional, una política monelaria contraccionista tiene efeclos inflacionarios (Spigelman 1987), en tanto que obliga a aumentar la demanda por créditos en el mercado financiero informal, con el consiguiente aumento de la tasa de interés y de los costos de las empresas. La falta de información sobre este fenómeno de los mercados financieros informales nos forzará, sin embargo, a utilizar la tasa de interés bancaria como indicador del costo del financiamiento de las empresas.

\section{El sector construcción}

Un sector que ha contribuido considerablemente a la inflación ha sido el sector consirucción. Ciertamente, la tierra urbana es un elemento extremadamente escaso en el país y la rápida urbanización de las últimas décadas desarrolló presión sobre el precio de la tierra. A esle efecto causado por el exceso de demanda de tierra le introdujo más combustible la especulación de los anos setenta.

Los excesos de demanda habitacional de la década pasada también facilitaron la ampliación extremada de los márgenes de ganancia de los constructores. Era usual en estos anos que las familias se veian obligadas a negociar con las empresas sobre la base de un plano arquitectónico y que antes de recibir la vivienda se le aumentara el costo contratado. En este proceso los precios de las viviendas casi se cuadruplicaron entre 1971 y 1978.

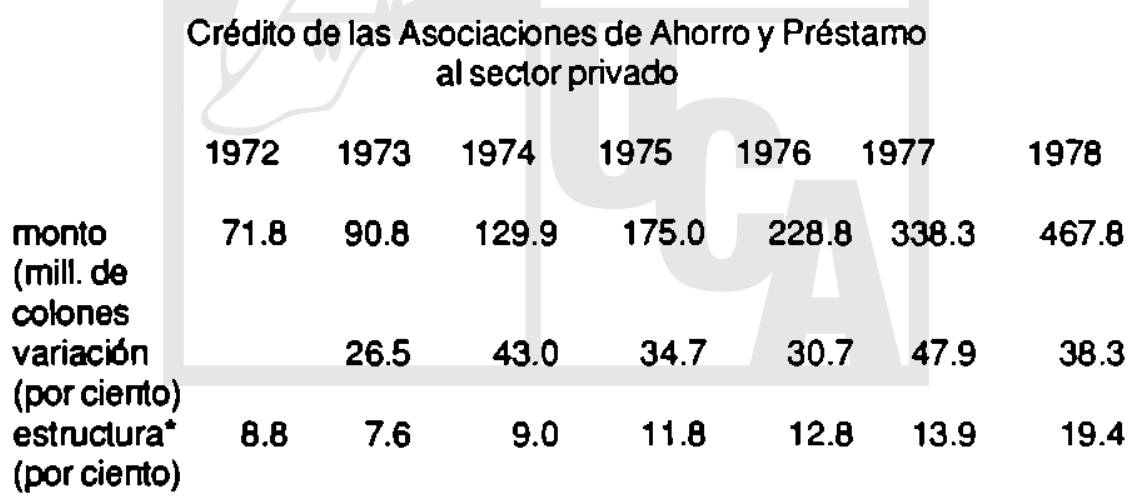

Fuento: Revistas del Banco Central de Reserva de El Salvador.

"Expresa la relación del crédito de las Asociaciones al sector privado al crédilo total de los Bancos Comerciales e Hipotecario. 
Un factor que facilitó este auge de la urbanización fue la política linanciera del sistema bancario. De 1972 a 1978 el crédito de las Asociaciones de Ahorro y Préslarno al seclor privado, en su mayor parte para construcción de vivienda, se multiplicó por 6.5 , pasando de 71.8 millones de colones a 467.8 millones.

El cuadro anterior muestra el dinamismo que adquirió el financiamienlo de la construcción en la década pasada. En eleclo, el coeficiente que expresa la relación del crédilo de las Asociaciones al crédilo tolal de los Bancos Comerciales e Hipotecario se duplicó en esios años. Obviamente la liquidez que resultó de los altos precios del calé a principios de la segunda mitad de la década conlribuyó considerablemente a facilitar esle auge.

Debe destacarse que en esta investigación el papel inflacionario del crédito bancario se restringe al campo de la construcción, porque es en este ámbito donde se encuentra que el exceso de demanda que provoca la expansión del crédito se enirenta a un factor escaso no producible ni importable, la tierra. Ciertamente este factor lambién es limilado en la producción de alimentos, pero, contrario al caso de viviendas, estos pueden ser importados cuando existen excesos de demanda.

En resumen, el factor principal que ha determinado el comportamiento de los precios de la construcción ha sido, además de los coslos de los insumos, la demanda excesiva de viviendas que impulsó, alimentada por la especulación, la elevación del precio de la tierra y la obtención de elevados márgenes de ganancia.

Los argumentos desarrollados hasta acá han constituido un primer apoyo empirico a nuestra hipótesis principal de que el proceso intlacionario ha estado determinado por el crecimiento de los costos relevantes, por las expectativas inflacionarias y por la expansión del sector consirucción. En la próxima sección sobre la base de estos argumentos elaboraremos un sencillo modelo de inflación por costos y lo someteremos en seguida a la evidencia econométrica.

\section{Un modelo de Inflación por costos}

La evidencia preliminar nos ha señalado que el criterio dominanle de lijación de precios en la economía salvadorefia es el de agregar un margen de ganancia a los costos. Un sencillo modelo puede ser elaborado sobre la base de este principio.

Llamemos:

$$
\begin{aligned}
& c=\text { costo unitario } \\
& w=\text { salario por unidad de tiempo }
\end{aligned}
$$




$$
\begin{array}{ll}
\pi & =\text { productividad del trabajo } \\
\mathrm{i} & =\text { lasa de interés nominal } \\
\mathbf{1} & =\text { fracción del costo unitario que se financia con } \\
& \text { crédito bancario }(\leq 1) \\
\mathbf{q}_{m} & =\text { coeficiente de insumos importados } \\
\mathbf{q}^{\mathbf{a}} & =\text { ceeliciente de insumos domésticos agrícolas } \\
\mathbf{q}^{m} & =\text { coeficiente de insumos doméslicos } \\
& \text { manulacturados } \\
\mathbf{p}_{m} & =\text { precio de los insumos importados en moneda } \\
\mathrm{E} & =\text { extranjera } \\
\mathbf{P}^{\mathbf{a}} & =\text { precio de los insumos domésticos agricolas } \\
\mathbf{p}^{m} & =\text { precio de los insumos domésticos } \\
& \text { manufacturados }
\end{array}
$$

Prescindiendo de la depreciación del capilal fijo para simplificar, podemos expresa la función de cosios unitarios de la empresa manufacturera de la siguiente manera:

$$
c=(\omega / \pi)+i(f c)+q_{m} p_{m} E+q^{a} p^{a}+q^{m} p^{m} \quad-1
$$

definiendo el precio de los insumos manulaclurados como el costo unitario más un margen de ganancia, es decir

$$
\mathbf{p}^{\mathrm{m}}=\mathrm{ca} \quad-2
$$

donde a es el coeliciente de transformación de costo a precio $(>1)$, y después de algunas Iransformaciones algebraicas (ver apéndice), la expresión 1 se convierte en:

$$
\begin{aligned}
\dot{c}= & \left(c f i /\left(\omega / \pi+q_{m} p_{m} E+q^{a} p^{a}\right)\right) \hat{i}+ \\
& (\omega / \pi) /\left(\omega / \pi+q_{m} p_{m} E+q^{a} p^{a}\right)(\omega / \tilde{\pi})+ \\
& \left(q_{m} p_{m} E\right) /\left(\omega / \pi+q_{m} p_{m} E+q^{a} p^{a}\right)\left(p_{m} E\right)+ \\
& \left(q^{a} p^{a}\right) /\left(\omega / \pi+q_{m} p_{m} E+q^{a} p^{a}\right)\left(\dot{p}^{\alpha}\right)+ \\
& \left(q^{m} p^{m}\right) /\left(\omega / \pi+q_{m} p_{m} E+q^{a} p^{a}\right) a .
\end{aligned}
$$

donde el símbolo " sobre una variable representa su lasa de variación. 
La ecuación de lasa de crecimiento de los costos nos permite deslacar tres aspectos:

1. que los coeficientes que acompanan a las variables independientes son ponderaciones.

2. que la auto alimentación de la producción manulacturera como insumos (producción de mercancías por medio de mercancías) introduce el margen de ganancia como variable explicativa de los costos; y

3. que el ponderador adecuado es el costo total unitario menos los costos del financiamiento y de los insumos manulaclurados; solamente cuando la producción no es financiada con crédito y no existe retroalimentación en la manyfactura el ponderador correspende al cosio lotal unitario.

Transformando los costos a precios, combinando las ecuaciones 2 y 3 y llamando $c^{*}$ al ponderador de los costos, obtenemos:

$$
\begin{aligned}
\hat{p^{m}=} & \left(c \mathrm{f} i c^{*}\right) \hat{\imath}+\left((\omega / \pi) / c^{*}\right)(w / \pi)+ \\
& \left(q_{m} p_{m} E / c^{*}\right)\left(\hat{p_{m}} E\right)+\left(q^{\theta} p^{a} / c^{*}\right) \hat{p^{a}}+\left(1+q^{m} p^{m} / c^{*}\right) \hat{a}
\end{aligned}
$$

la cual puede expresarse como sigue:

$$
\hat{p}^{m}=\alpha_{1} \hat{i}+\alpha_{2}(\omega \dot{j} \pi)+\alpha_{3}(\dot{p} \dot{m} E)+\alpha_{4} \dot{p^{a}}+\alpha_{5} \hat{a}
$$

Vamos a definir el índice de coslo de vida, $P$, como la ponderación de tres índices: precios agrícolas no exporlables, precios de bienes manufacturados y precios de la construcción; es decir:

$$
\hat{\mathrm{P}}=\gamma_{1} \dot{\mathrm{p}}^{\omega}+\gamma_{2} \dot{\mathrm{P}}^{m}+\gamma_{3} \dot{\mathrm{P}}^{\mathrm{c}}
$$

donde $\bar{p}^{c}$ es la lasa de crecimienlo de los precios de la construcción

Sustituyendo 4 en 5 y ordenando se obtiene:

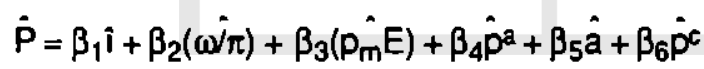

La ecuación anlerior debe modificarse adicionalmente conforme a los argumentos que desarrollamos en la primera sección:

1. Los precios agricolas eslán determinados por los costos, por los precics de garantía y por los precios de las imporlaciones internacionales lo cual nos permite eliminar la tasa de variación de los precios agrícolas 
puesto que su electo estaría considerado en los restantes términos;

2. El margen de ganancia se ajusta según las expectativas inflacionarias que se forman los encargados de fijar los precios; adoptaremos para tal efecto la hipólesis de expectativas adaptativas; $y$

3. Los precios de la consirucción dependen del crecimiento del sector.

En consecuencia la ecuación para estimar será la siguiente:

$$
\begin{aligned}
& \hat{\mathbf{P}}=\beta_{1} \hat{\imath}+ \beta_{2}(\hat{\omega} / \pi)+\beta_{3}\left(\hat{p}_{m} E\right)+\beta_{5} \dot{p}^{\theta}+\beta_{6} \hat{y}_{c} \\
& \text { donde: } \quad \hat{p}^{\theta}=\text { inflación esperada } \\
& \dot{y} c=\text { crecimiento del sector construcción }
\end{aligned}
$$

Previamenle a la estimación empirica del modelo debemos resolver dos problemas: debemos encontrar la lorma de cuantificar la productividad del trabajo y las expeclativas inflacionarias.

Aunque variable clave para establecer las políticas de desarrollo, a la productividad no se le da seguimiento oficial y por tal razón no se le encuentra en ningún tipo de iníorme. Como el modelo sefala, sin embargo, juega un papel importanle en el proceso inflacionario y en consecuencia habrá que intentar lograr un estimado previo de su evolución.

El cálculo directo a través de su propia delinición, dividiendo la producción por el empleo, se frustra por la inexislencia de información sobre esla última variable macroeconómica. En consecuencia la estimación tendrá que ser indirecta; el mélodo a seguir tiene su origen en la teoría y en la evidencia empírica. Se trala de aplicar la "Ley Verdoorn". Esta ley empírica pero con lundamento teórico, fue lormulada primeramente por Verdoom (Verdoom 1949) y replanteada por Nicholas Kaldor ${ }^{7}$ de la Universidad de Cambridge en 1967. La ley destaca el papel de los rendimientos crecientes a escala en la industria. Establece que asociado al crecimiento industrial se encuentra el crecimiento de la productividad del trabajo. Más concretamente establece que entre más rápidamente crece la producción de la industria más rápidamente crecerá la productividad. Funcionalmente puede plantearse de la siguiente manera:

$$
\hat{\pi}=f(\hat{y})
$$

El fundamento teorico se remonta hasta Adam Smilhe. En los primeros 3 capílulos de la Riqueza de las Naciones, Smith argumenló que a medida que se expande la producción, lo cual depende de la extensión 
del mercado, se prolundiza la división del trabajo y ésta impulsa la productividad (el rendimienlo por unidad de trabajo en el lenguaje de Smith). El impulso que recibe la productividad proviene del hecho que la especialización de la división del trabajo provoca mayor habilidad técnica, mayor inventiva, nuevos conocimientos que se retroalimentan a través de los procesos de aprendizaje (learning by doing), etc.

Macroeconómicamente, con el crecimiento de la industria muchas economias de escala surgen del desarrollo de la diferenciación y de nuevas industrias subsidiarias, así como de la absorción de nuevas tecnologias?.

Empíricamente, Kaldor eslimó esta relación en 12 países induslriales con variadas tasas de crecimienlo entre 1953 y 1964 (Kaldor 1967). EI resultado que obtuvo fue el siguiente:

$$
\dot{\pi}=1.053+0.484 \hat{y} \quad \mathrm{R}^{2}=0.826
$$

La ecuación es estadisticamente significaliva y sugiere fuertemente en el caso analizado que el crecimiento del producto jugó un papel importante en la determinación del crecimiento de la productividad.

Una formulación variante de esla ley distingue entre el crecimiento tendencial del producto manufacturero y el crecimiento cíclico alrededor de la tendencia de largo plazo que resultan de los cambios en la capacidad instalada ociosa. El modelo se especifica de la siguiente manera:

$$
\begin{aligned}
& \ln \pi=\alpha_{0}+\alpha_{1} \ln y^{\prime}+\alpha_{2} \ln y^{v} \\
& \text { donde: } \quad y^{\prime}=\text { lendencia de largo plazo del producto } \\
& y^{*}=\text { variaciones de corto polazo del producto }
\end{aligned}
$$

También este modelo fue verificado empiricamente con anterioridad (Vásquez 1981). De 208 eslimaciones realizadas en México correspondientes a igual número de subgrupos industriales, el $60 \%$ presenló un coeticiente de determinación corregido superior a 0.80 y $75 \%$ superior a 0.60 . El coeficiente de regresión $\alpha_{1}$ resultó significativo y positivo en alrededor de $75 \%$ de los casos y el coeliciente $\alpha_{2}$ en $62 \%$.

Este último modelo es el que nos servirá de fundamento para la estimación de la productividad en El Salvador. El problema que se presenta es que solamenle se dispone de 4 datos de la produclividad conforme a los Censos Industriales de 1951, 1961, 1971 y 1978. La intrapolación y la 
extrapolación se harán en tres etapas. Primeramente estimamos la lendencia del producto industrial con 37 datos mediante la expresión siguiente en el periodo 1950-1986:

$\log y$ mat $=a+b t$

donde: $y$ maf $=$ producto manulacturero a precios constamles

$$
\begin{aligned}
& \text { de } 1982 \\
& l=\text { tiempo } \\
& b=\text { estimado de la tasa tendencial de crecimiento }
\end{aligned}
$$

El resultado que se obtuvo fue el siguiente:

$$
\begin{array}{lll}
\text { logymal }=\begin{array}{l}
4.75 \\
(32.6)
\end{array}+\begin{array}{l}
0.05 t \\
(7.8)
\end{array} & \mathrm{R}^{2}=0.63 \\
\mathrm{D}-\mathrm{W}=1.46
\end{array}
$$

En seguida definimos el producto de corto plazo de la manulactura (ymar) como el cociente entre el producto corriente y el producto tendencial calculado por medio de la ecuación de tendencia recién estimada y se procede a aplicar la ley Verdoorn para el número reducido de dalos disponibles. El resultado se muestra a continuación:

$$
\begin{aligned}
& \log \pi=\underset{(81.9)}{6.28}+\underset{(40.7)}{0.55 \operatorname{logymaft}}+\underset{(6.4)}{1.13 \operatorname{logymaN}} \\
& \mathrm{R}^{2}=0.99
\end{aligned}
$$

El elevado valor de $R^{2}$ no debe confundir, pues se debe al número reducido de datos utilizados. Cierlamente esta es una gran limilación del mélodo aplicado, pero permite una primera aproximación de la variable requerida. La intrapolación y extrapolación necesarias procede, entonces, de esta ecuación eslimada sustiluyendo los sucesivos valores de los ingresos tendencial y cíclico. La serie estimada se presenta en el apéndice.

El segundo problema que hay que resolver es el de las expectativas; para éslo utilizaremos las expeclalivas adaptativas ${ }^{10}$. La hipótesis afirma que la inllación que en el año $t-1$ esperamos que ocurra en el año $t$, es igual a la que en el afio t-2 se esperaba que ocurriera en el año t-1 corregida en una cantidad proporcional al error comelido en el período anterior. Formalmente se expresa de la siguiente manera:

$$
\hat{p}_{t}^{w}=\dot{p}_{t-1}{ }^{w}+\beta\left(\dot{p}_{t-1}-\dot{p}_{t-1}^{\theta}\right)
$$


El modelo de expectalivas es facilmemte convertible en uno de rezagos distribuidos.

$$
\dot{p}_{t}^{\theta}=\beta \hat{p}_{t-1}+\beta(1-\beta) \hat{p}_{1-2}+\ldots
$$

No podemos sustituir directamenle esta expresión en la ecuación de coslos que queremos estimar, pues nos introducirla problemas en la estimación por el método de minimos cuadrados ordinarios, ya que la misma variable dependiente rezagada aparecería como explicaliva ${ }^{11}$. Para evitar este problema estimaremos previamente la inflación esperada aplicando el método de la minimización de los errores de pronóstico ${ }^{12}$. El problema se plantea así: ¿cuál es el valor del parámetro $\beta$ que minimiza el error comelido por los agentes economicos cuando se forman sus expectalivas? Formalmente:

$\min . \sum\left(\hat{p}_{t}-\dot{p}_{t}^{\theta}\right)^{2}=\sum_{t=1}^{n}\left(\dot{p}_{t}-\beta \dot{p}_{t-1}-\beta(1-\beta) \dot{p}_{t-2 . .}\right)^{2}$

Reduciendo los rezagos a 3 , dándole valores sucesivos a $\beta$ a partir de cero en incrementos de 0.1 y luego de 0.01 , obluve los resultados siguientes:

$\begin{array}{lrrrrrr}\text { parámelro } & 0.9 & 1.0 & 1.1 & 1.15 & 1.16 & 1.17 \\ \text { error } & 431.7 & 426.4 & 424.8 & 424.6 & 423.6 & 424.6\end{array}$

Como muestran los resultados, el valor del parámetro que minimiza los errores es 1.16, este valor aunque mayor que uno permite la convergencia del modelo ${ }^{13}$... Cuando el valor del parámetro $\beta$ es mayor que uno y menor que dos la inllación esperada converge a la inflación del periodo anterior. Con este cálculo se obtuvo la serie de expectalivas inflacionarias la cual se muestra en el apéndice.

Antes de estimar el modelo de costos propuesto es necesario hacer tres aclaraciones más sobre la inlormación utilizada. La tasa de interés que lorma parte de los coslos de producción y que debería de considerarse en este análisis, tendria que ser una ponderación de las tasas oficial y del mercado financiero informal. Por falta de información, consciente de que se subestimará el resultado, se ulilizará la oficial preferencial clase A para la industria.

Como indicador del salario nominal se ulilizará el salario mínimo para la industria. En vista que los aumentos de salario no se dan a principio de ano, durante un mismo ano pueden coexistir varios niveles de salario; por esta razón el indicador de salario utilizado será un promedio de cada año. 
Finalmente, el indicador del precio de las importaciones será un Indice ponderado de los precios de los 5 principales socios comerciales de El Salvador (USA, Alemania, Japón, Guatemala y Costa Rica) por la estructura de las importaciones correspondientes.

He estimado dos versiones del modelo de coslos. En el primero he incluido la variable productividad entre las variables explicalivas como parte del costo salarial unitario; en el segundo la he excluido y he dejado que su efecto se haga sentir a Iravés del componente estocáslico. Las razones de proceder de esla manera se encuentran en el origen indirecto de la información sobre la productividad. Las estimaciones econométricas utilizando mínimos cuadrados ordinarios en el periodo 1971-1986 se muestran a continuación, donde los coelicientes entre paréntesis indican los estadístico $\mathrm{t}$.

\section{Version 1.}

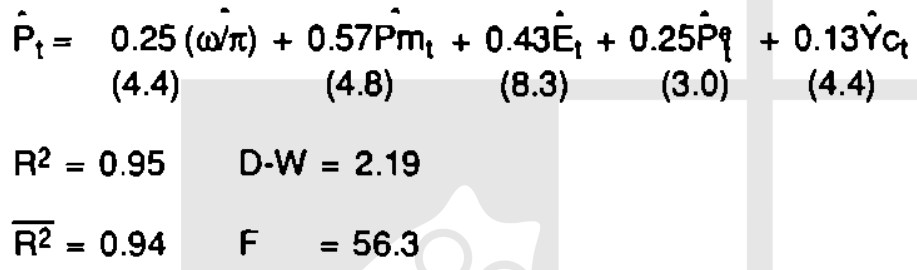

Versión 2.

$$
\dot{\mathrm{P}}_{\mathrm{t}}=\underset{(2.9)}{0.24 \dot{\omega}_{\mathrm{t}}}+\underset{(2.7)}{0.58} \dot{\mathrm{P}}_{\mathrm{t}}+\underset{(3.2)}{0.39 \hat{\mathrm{E}}_{\mathrm{t}}}+\underset{(3.9)^{1}}{0.32 \hat{\mathrm{P}}^{\theta}}+\underset{1}{0.09)} \dot{\mathrm{Y}}_{\mathrm{t}}
$$

$$
\begin{array}{rlrl}
\mathrm{R}^{2} & =0.93 & \mathrm{D} \cdot \mathrm{W} & =1.90 \\
\overline{\mathrm{R}}^{2} & =0.90 & \mathrm{~F} & =35.3
\end{array}
$$

Ambos resultados son garantes de la robustez del modelo de inflación por costos ${ }^{14}$; los dos coeficientes de determinación indican que las dos versiones son capaces de dar cuenta de más del $90 \%$ de las variaciones de la inflación. Los coeficientes $\mathbf{t}$ son estadísticamente significalivos al $1 \%$ excepto el estimador de la tasa de crecimiento de la construcción que en la segunda versión lo es al $2 \%$. Un resullado importante de comentar es el referente al Durbin-Watson. Según el resullado de la primera versión ( $D-W=2.19$ ) no se puede concluir si hay o no aulocorrelación pues se ubica en la zona de indecisión, aunque muy cerca de la frontera de no aulocorrelación. La segunda versión es superior en este aspecto ya que el Dußin-Watson muestra ausencia de autocorrelación (D-W = $1.90)$. 
Por los resultados anteriores hemos escogido la segunda versión del modelo de costos para presentar en los siguientes grálicos la inflación estimada comparada con la real y el peso inflacionario respectivamente. El modelo como se ve en el primer gráfico reproduce con notable precisión el proceso inflacionario real, tanto sus momentos de aceleración como los de amortiguamiento. El repunte de los últimos anos es caplurado por el modelo casi sin ningún rezago. Por otra parte, el segundo grático nos

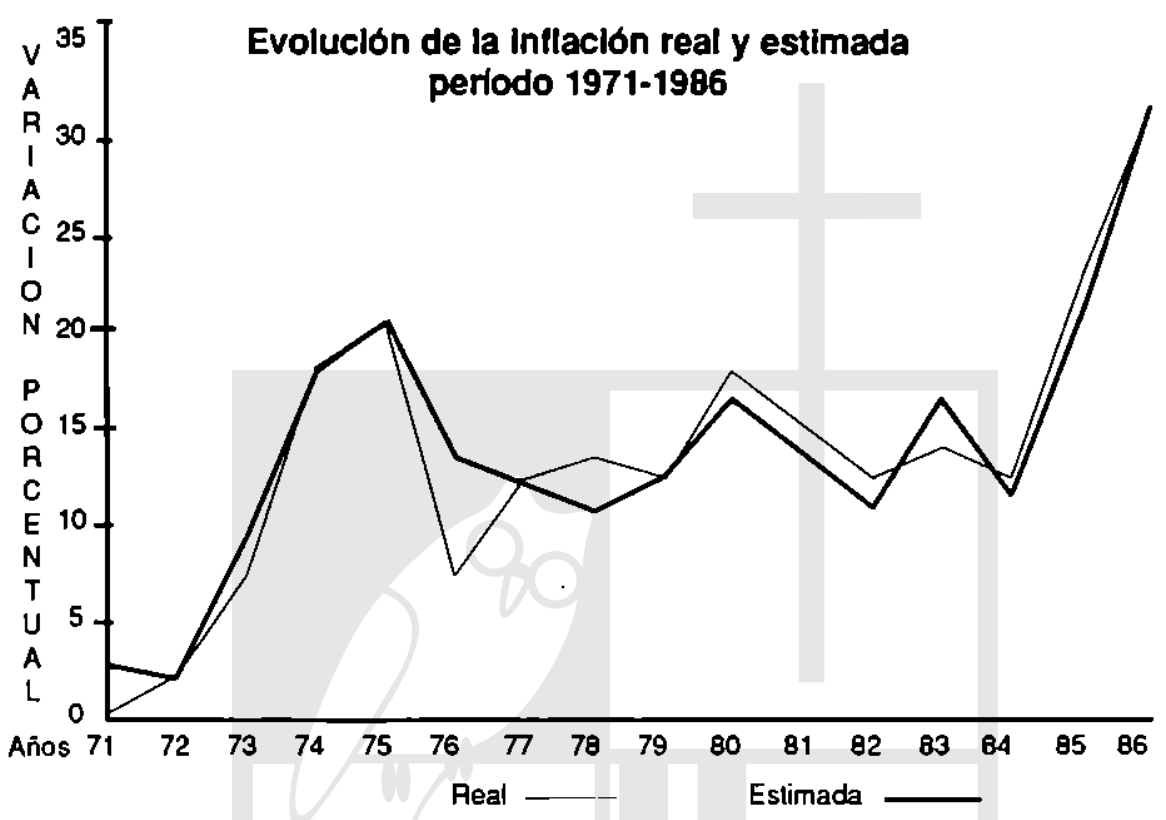

muestra que el factor dominante de la inflación en la década pasada fue el precio de las importaciones; mientras que en el repunte reciente ha sido la devaluación progresiva del lipo de cambio. La composición inflacionaria nos permite también mosirar la poca contribución que el coslo del trabajo ha tenido en el proceso inflacionario. En electo, el salario solamente aportó en $19745.7 \%$ y en $19807.5 \%$, el resto de los anos lo ha hecho a niveles inferiores a 3.5\%. Observese asimismo el papel importante de las expeclativas inflacionarias como factor inercial en el proceso inflacionario. También es imporlante notar el impacto inflacionario del sector consIrucción en 1975 y 1977, precisamente los af́os de auge constructivo y de expansión de las Asociaciones de Ahorro y Préstamo.

Finalmente es importante destacar las elasticidades eslimadas. La ecuación estimada nos muestra que un aumento de uno por ciento en 
Peso Inflaclonarlo periodo 1971-1978

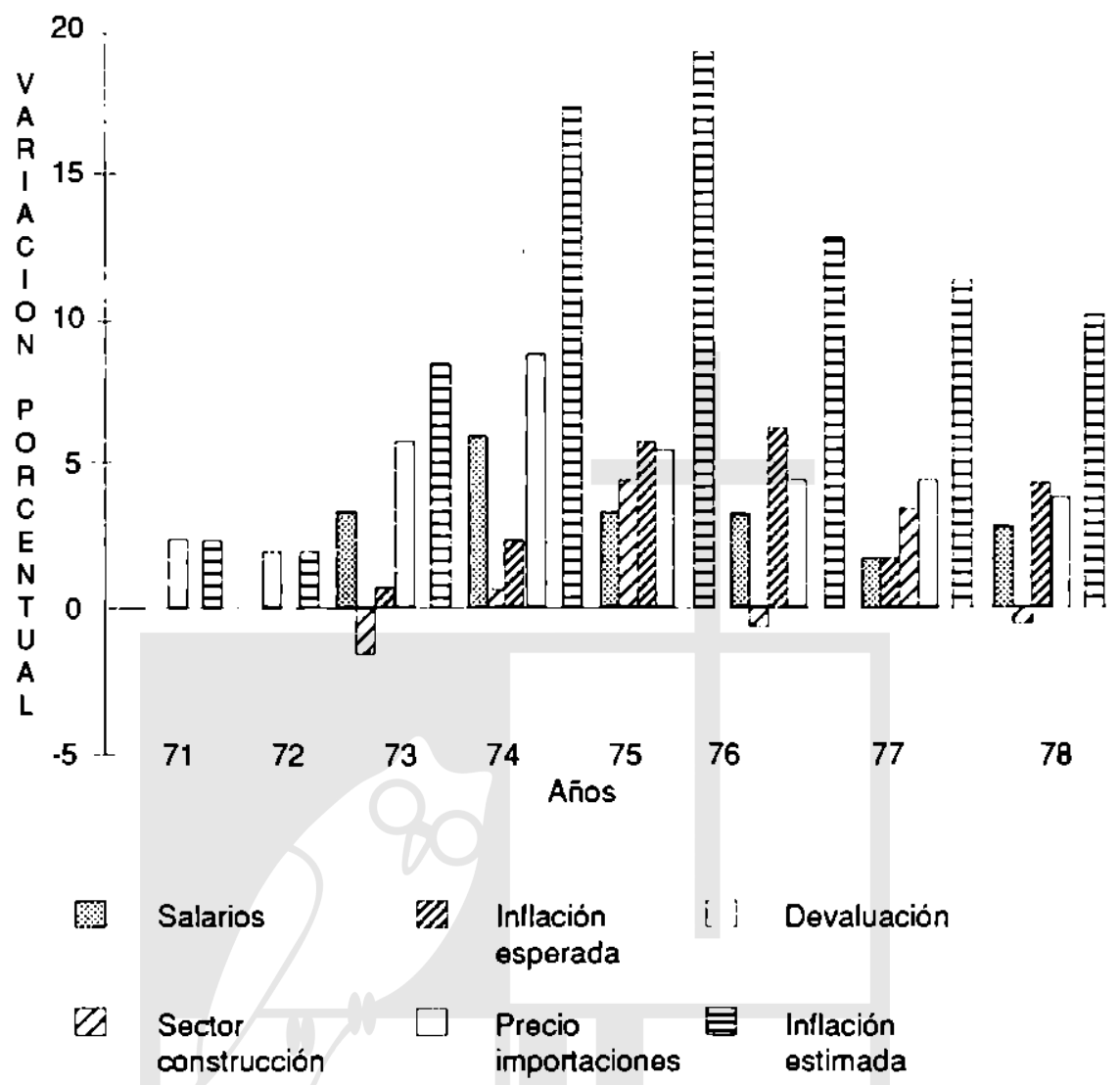

los salarios nominales sin cambios en la productividad, impulsarian $0.24 \%$ de inflación. Por el contrario, si a la par que se incrementan los salarios se logra un aumento proporcional en la productividad, el efecto inflacionario seria nulo. Muy importante para la problemática aclual es el impacto de la devaluación sobre la inflación. Observese que por cada punto de devaluación se impulsa casi medio punlo de inllación (0.39). Otro indicador muy importanle es el ajusle en las expectativas; conforme a la lógica de nuestra argumentación microeconómica de formación de precios, el resultado indicaria que los empresarios han estado logrando trasladar por cada punlo de inflación esperada, un lercio de punto a los precios. La conclusión es razonable, pues como ya hemos indicado, el contexto salvadorefio es oligopólico, lo cual contribuye a que el sector empresarial se proteja con relativa facilidad de la ińlación. 


\section{Peso Inflaclonarlo período 1979-1986}

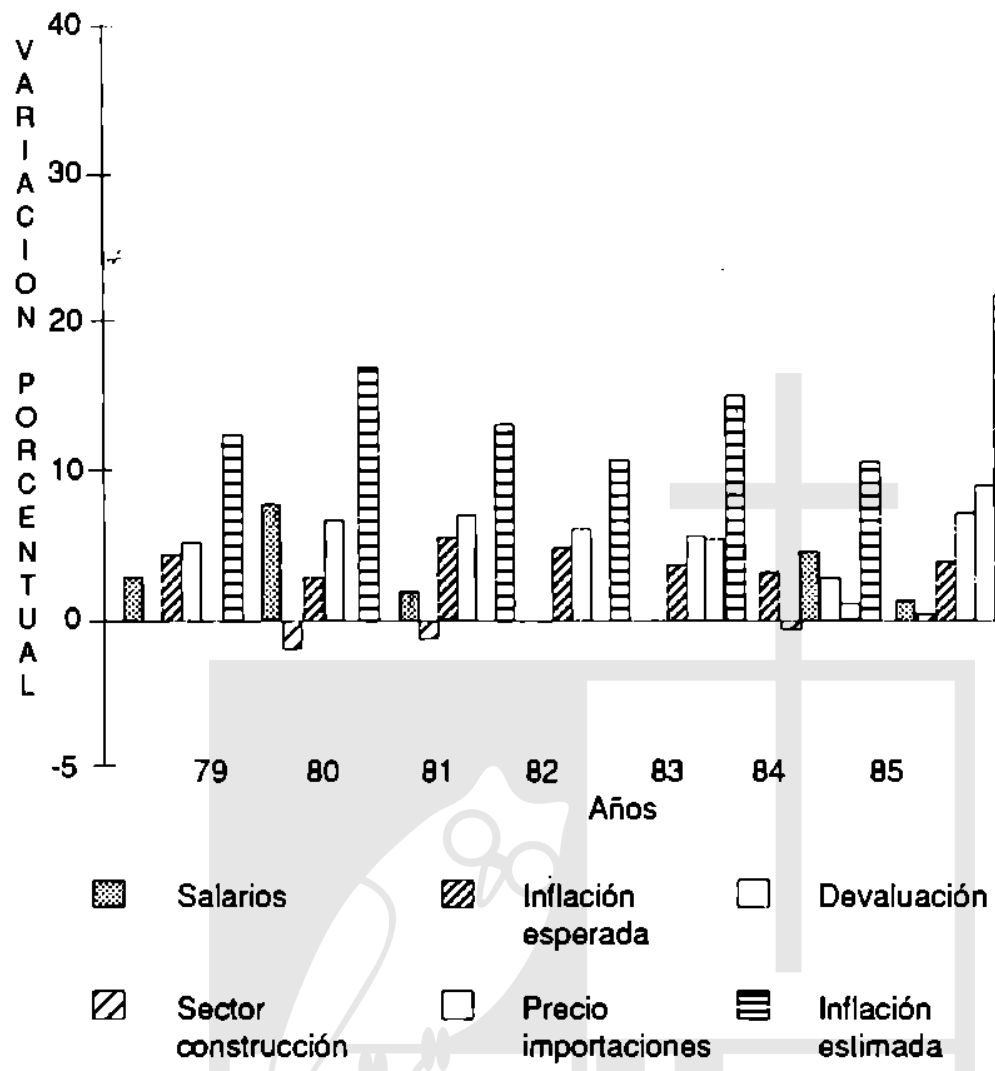

III. Modelos monetarlstas de la Inflaclón

En la primera parte de esla sección se presentan los fundamentos de algunos modelos monetarislas de la inflación que han sido aplicados en El Salvador y en América Latina. En la segunda parte, estos modelos se somelen a la prueba econométrica en el período reciente.

Los primeros dos modelos tienen su raiz en el modelo monetarista de conomia cerrada que Arnold Harberger aplicó a la economía chilena a principios de los ańos sesenta (Harberger 1963). La base de este modelo es la leoria cuantitativa del dinero:

$$
P=M C N
$$


donde: $P=$ nivel general de precios

$M=$ olerta monetaria

$\mathrm{C}=$ velocidad de circulación del dinero

$Y=$ producto real

Expresada en tasas de crecimiento:

$$
\dot{P}=\dot{M}+\dot{C}-\dot{Y}
$$

es decir, la inflación depende directamente del crecimiento de la oferta monelaria, de los cambios de la velocidad de circulación del dinero e inversamente del crecimiento del producto.

Sobre esta base, Harberger, cubriendo el período 1939-1958, eslimó el siguiente modelo:

$$
\hat{P}_{t}=a_{0}+a_{1} \hat{Y}_{t}+a_{2} \dot{M}_{t}+a_{3} \hat{M}_{l-1}+a_{4} \dot{P}_{t}+a_{5} \dot{W}_{t}
$$

donde: $\hat{\hat{P}}_{\mathrm{t}}=\dot{\mathrm{P}}_{\mathrm{t}-1}-\dot{\mathrm{P}}_{\mathrm{t}-2}$

$$
\dot{W}_{1}=\text { lasa de variación de los salarios nominales }
$$

Harberger incluye la variación de la oferla monetaria rezagada un período, asumiendo que el impaclo del dinero sobre los precios se desarrolla plenamente en dos años. Asimismo incluye la aceleración de la inflación, $p_{1}$, como medio para tomar en cuenta las variaciones en la velocidad de circulación monetaria, a través del costo de oportunidad del dinero. El componente estructural de la inflación es tratado de caplurar por medio de los salarios.

El resullado que Harberger obluvo fue el siguiente:

$$
\begin{aligned}
& \dot{\mathrm{P}}_{\mathrm{t}}=-0.32+0.74 \dot{\mathrm{M}}_{\mathrm{t}}+0.34 \dot{\mathrm{M}}_{\mathrm{t}-1}-0.91 \dot{\mathrm{Y}}_{\mathrm{t}}+0.20 \dot{\mathrm{P}}_{\mathrm{t}} \\
& \quad(-0.03) \quad(4.63) \quad(.2 .27) \quad(-2.94) \quad(1.67) \\
& \mathrm{R}^{2}=0.87
\end{aligned}
$$

La ecuación ajustó estadísticamente bien, exceptuando el término constante y los salarios que no fueron estadisticamenle significativos y la aceleración de la ińlación que sólo lo fue al $5 \%$, lodos los parámetros resultaron según las expeclativas del autor. Obsérvese que el resultado econométrico mostró que cierlamente el impaclo del dinero se desarrolla en dos afos $\left(a_{2}+a_{3} \approx 1\right)$. 
Una ampliación del modelo anterior fue el elaborado por J. Nugent y C. Glezakos (1979). El modelo supera al de Harberger en tres aspectos:

1. Es un modelo simultáneo de determinación de la inflación y el ingreso;

2. Incomora las expectativas de los agentes económicos; y

3. Distingue entre ajustes de corto y largo plazos, descomponiendo el ingreso real en ingreso permanente e ingreso transitorio.

Las ecuaciones propuestas por eslos autores son las siguientes:

a. Ecuación de olerta:

$$
Y_{t}=Y_{t}+F\left(P_{t} / P_{t}^{\theta}\right)
$$

donde el primer componente es el ingreso permanente y el segundo el ingreso Iransilorio, el cual es función de la relación entre el precio real y el precio esperado 15 .

Expresada en lasas de cambio y agregando el tipo de cambio real corriente y rezagado un periodo, la anterior ecuación se convierte en:

$$
\dot{Y}_{t}=\alpha_{1} \hat{Y}_{t}{ }^{\theta}+\alpha_{2}\left(\dot{P}_{t}-\dot{P}_{t}^{\theta}\right)+\alpha_{3}\left(\dot{E}_{t}-\dot{P}_{j}\right)+\alpha_{4}\left(\dot{E}_{t-1}-\dot{P}_{t-1}\right)-1
$$

en donde se espera que:

$$
\alpha_{1}=1 ; \alpha_{3}, \alpha_{4}>0
$$

b. Ecuación de precios:

el modelo utiliza la misma ecuación de precios del modelo de Harberger con algunas modificaciones.

$$
\dot{\mathrm{P}}_{\mathrm{t}}=\gamma_{1} \hat{\mathrm{M}}_{1}+\gamma_{2} \dot{\mathrm{M}}_{\mathrm{t}-1}+\gamma_{3} \dot{\mathrm{Y}}_{\mathrm{t}}^{\theta}+\gamma_{4} \dot{\mathrm{Y}}_{\mathrm{t}}^{\mathrm{v}}+\gamma_{5} \dot{\mathrm{E}}_{\mathrm{t}}+\gamma_{6} \dot{\hat{\mathrm{P}}}_{\mathrm{t}}^{\theta}-2
$$

donde se espera, según la teoria que:

$$
\begin{aligned}
& 0 \leq \gamma_{1} ; \gamma_{2} \leq 1 ; \gamma_{3}, \gamma_{4} \leq 0 ; \gamma_{5} \geq 0 ; \gamma_{6} \geq 0 \\
& \hat{Y}_{t}=\text { tasa de variación del ingreso esperado; } \\
& \hat{Y}_{t}^{v}=\text { tasa de variación del ingreso transilorio; } \\
& E_{t}=\text { lipo de cambio; } \\
& \hat{P}_{t}^{\theta}=\text { inflación esperada. }
\end{aligned}
$$


Aplicando el método de cálculo de expectalivas adaplativas que aplicamos en la sección anterior, los aulores estimaron el modelo en el período 1950-1969 en 16 paises latinoamericanos, utilizando mínimos cuadrados bi-etápicos. El resultado se muestra en el siguienle cuadro:

$$
\begin{aligned}
& \text { A. Paises con inflación alta } \\
& \dot{\mathrm{P}}_{\mathrm{t}}=0.545 \dot{\mathrm{M}}_{\mathrm{l}}+0.308 \dot{\mathrm{M}}_{\mathrm{t}-1}-1.108 \hat{\mathrm{Y}}_{\mathrm{t}}^{\mathrm{\theta}}-0.229 \dot{\mathrm{Y}}_{\mathrm{t}}^{\mathrm{v}}+0.109 \hat{\mathrm{E}}_{\mathrm{t}}+ \\
& \begin{array}{llll}
\text { (8.6) } \quad(5.1) \quad(-1.4) \quad(-0.6) & 0
\end{array} \\
& 0.253 \hat{\bar{P}}_{\mathrm{t}} \\
& \mathrm{R}^{2}=0.794 \\
& \text { B. Países con inllación mediab } \\
& \dot{P}_{t}=-0.099 \dot{M}_{t}-0.364 \dot{M}_{t-1}+3.943 \hat{Y}_{t}^{\theta}+1.390 \hat{Y}_{t}^{v}+0.122 \dot{E}_{t}- \\
& \begin{array}{llll}
(-0.3) & (-0.7) \quad(1.2) \quad(0.9)
\end{array} \\
& 0.725 \hat{\hat{P}}_{\mathrm{t}} \mathrm{e} \\
& (-0.7) \\
& \mathrm{R}^{2}=0.0 \\
& \text { C. Paises con inflación bajac } \\
& \dot{P}_{t}=-0.404 \dot{M}_{t}+0.074 \dot{M}_{t-1}+1.110 \dot{\gamma}_{t}^{\theta}+1.621 \dot{\gamma}_{l} 0.147 \dot{E}_{t}+ \\
& (-1.4) \\
& \text { (0.7) } \\
& \text { (1.4) }(-0.4) \\
& 0.456 \hat{\bar{P}}_{\mathrm{t}}^{\theta} \\
& \mathbf{R}^{2}=0.0
\end{aligned}
$$

a. Argentina, Bolivia, Brasil, Chile y Unuguay. $\dot{\mathrm{P}}>20 \%$ 
b. Colombia, México, Paraguay y Perú. $5<\dot{P}<20$

c. Costa Rica, Ecuador, El Salvador, Gualemala, Honduras, Nicaragua y Venezuela. $\dot{P}<5$

Los resullados revelan que el modelo lunciona adecuadamente sólo de manera parcial en los paises con inflación alta. Decimos parcialmente, pues aun en este caso los coeficientes del ingreso permanente y del transitorio no fueron estadisticamente significativos. No obstante los coeficientes de la olerta monelaria responden igual que los obtenidos por Harberger; es decir, el impacto de la oferta monelaria se desarrolla casi completamente en dos anos.

En contrasie, los resullados obtenidos en los otros dos grupos de países fueron desastrosos. Ningún coelicienle fue esladísticamente signilicalivo y ambas bondades de ajusle lueron nulas; 10 cual, sefalan los autores, no es sorprendente en el caso de los países con inflación baja pues hay muy poca variación que explicar; pero si lo es en el otro caso de inflación media.

Los autores terminan sefialando entre otras cosas que "los intentos de explicar o de predecir el comportamiento de corlo plazo en los paises menos desarrollados con teorias basadas sobre caracteristicas institucionales y socioeconómicas de los países desarrollados o de relaciones de largo plazo como las de los modelos monetarislas de la inflación no siempre pueden tener éxito".

El último modelo que vamos a presentar es el del enfoque monetario de la balanza de pagos (Frenkel y Johnson 1976, International Monetary Fund 1977). Desde el punto de vista monetarisla, éste es el tipo de modelo más apropiado para una economia pequef́a y abierta como la salvadorena.

La primera hipotesis de este enfoque es que un desequilibrio en el mercado monetario se refleja en la balanza de pagos. Asi, un exceso de crecimienlo del crédito doméstico sobre el crecimiento de la demanda de dinero se Iraduce en la adquisición de activos del exterior deteriorando la balanza de pagos.

La segunda hipólesis, es la referenle a los precios y se conoce como la ley de un solo precio. El precio doméslico se delermina según el precio internacional. Se comienza distinguiendo enlre bienes que forman parte del comercio intemacional (Iradeable goods o bienes comerciables) y bienes que sólo se comercian al interior del pais (bienes no comertiales). El Indice general de precios resulta en consecuencia de la ponderación de ambos tipos de bienes: 


$$
P=P_{c}^{a} P_{n c}(1-\alpha)
$$

donde $\quad P=$ indice general de precios del pals

$P c=$ índice de precios de los bienes no comerciables

En tasa de variación la expresión se convierte en:

$$
\hat{\mathrm{P}}=\alpha \hat{\mathrm{P}}_{\mathrm{c}}+(1-\alpha) \hat{\mathrm{P}}_{\mathrm{nc}}
$$

donde: $\alpha<1$

Tratándose de una pequena economía abierta, el precio de los bienes comerciables tiene que ser igual a los precios intemacionales y los precios de los bienes no comerciables, aunque pueden dilerir en el corto plazo de los internacionales, por los electos sustitución en el consumo y en la producción, en el largo plazo tienden a ser iguales. Sustituyendo en 2:

$$
\begin{aligned}
& \dot{\mathrm{P}}=\alpha \dot{\mathrm{PI}}+\alpha \dot{\mathrm{E}}+(1-\alpha) \dot{\mathrm{PI}}+(1-\alpha) \dot{\mathrm{E}} \\
& \dot{\mathrm{P}}=\dot{\mathrm{PI}}+\dot{\mathrm{E}}
\end{aligned}
$$

donde: $\mathrm{PI}=$ precio internacional

$E=$ tipo de cambio

La ley de un solo precio es entonces un resultado de largo plazo. La inflación en el largo plazo no puede diferir de la inflación internacional. En el corlo plazo, sin embargo, la inflación doméslica puede diferir de la mundial, tanlo por el rezago en el ajuste de los precios de los bienes no comerciables, como por los excesos de crédito doméstico. Es este fenómeno el que tralan de evidenciar algunos trabajos como el de Mario Blejer (1978) en México y el de Luis de Sebastián (1986) en El Salvador. Veamos esle último.

De Sebastián poslula que en el corto plazo los precios domésticos poseen un componente exógeno y un componente endógeno el cual es función del exceso de oferta monetaria, es decir:

$$
P=P^{*}+b_{3} E O
$$

donde: $\quad E O=$ exceso de oferta de dinero

$$
\begin{aligned}
& P^{*}= \text { precio doméstico determinado exclusivamente } \\
& \text { por factores exógenos. }
\end{aligned}
$$


Para el precio exógeno el autor propone una tunción dinámica de la siguiente forma:

$$
P^{*}=b_{0}+b_{1} P I+b_{2}\left(b_{1} P I_{t-1}-P_{t-1}^{*}\right)
$$

La equación 4 pasa a ser en consecuencia:

$$
P_{t}=b_{0}+b_{1} P I_{t}+b_{2}\left(b_{1} P I_{t-1}-P_{t-1}\right)+b_{3} E O_{t} \quad-6
$$

Por otra parte, el equilibrio de la oferta y de la demanda de dinero exige que:

$$
h(R+D)=\left(P \cdot m^{d}\right)
$$

donde $\mathrm{R}$ = nivel de reservas internacionales en moneda nacional

$$
\begin{aligned}
& D=\text { nivel de crédito doméstico } \\
& \mathbf{m}^{\mathbf{d}}=\text { demanda de saldos reales. } \\
& \mathbf{h}=\text { multiplicador monetario. }
\end{aligned}
$$

El aulor introduce una variable, $D^{*}$, que la define como el slock de crédito doméstico que seria juslamente el necesario para satisfacer la demanda de dinero a un stock constante de reservas internacionales con un nivel de precios delerminado sólo por fuerzas exógenas, $P^{*}$. Definida asi $D^{*}$, si el crédito real fuera igual al nivel de $D^{*}$, este alcanzaría exactamente para satisfacer la demanda de dinero y ni la balanza de pagos se alteraría ni los precios domésticos diferirian del precio exógeno; pero si crece más allá de este nivel, aparecerá un exceso de oferta de dinero que incidirá negativamente en la balanza de pagos y presionará los precios al alza. Para poder definir $D^{*}$ es necesario lormular las siguientes dos restricciones:

$$
\begin{aligned}
& R_{1}=R_{t-1} \\
& P=P^{*}
\end{aligned}
$$

$D^{*}$ se puede expresar de la siguiente manera ${ }^{16}$ :

$$
D_{t}^{*}=P_{t}^{*}-R_{t-1}+m_{t}^{d}
$$

y en consecuencia:

$$
E O_{t}=D t-D_{t}^{*}=D_{t}+R_{t-1}-m_{t}^{d}-P_{t}^{*}
$$


sustituyendo, tomando primeras diferencias y expresando en logarilmos se obtienen las dos ecuaciones que servirán para estimar el modelo.

$\left(R_{t} A_{t}+D_{t}\right)\left(\Delta \log R_{t}-\Delta \log R_{t-1}\right)=\left(b_{3}-1\right)\left(D_{t} / R_{t}+D_{j} \Delta \log D_{t}+\right.$

$R_{f} /\left(R_{t}+D_{j}\right)\left(\Delta \log R_{t-1}-\Delta \log \pi{ }^{d} t\right)+b_{1}\left(1-b_{3}\right) \Delta \log P_{t}+$

$b_{2}\left(1-b_{3}\right) b_{1} \Delta \log P I_{t-1}-b_{2}\left(1-b_{3}\right) \Delta \log P_{t-1}$

$\log P_{t}-b_{1} \log P_{t}=b_{2}\left(1-b_{3}\right)\left(b_{1} \log P_{t-1}-\log P_{t-1}\right)+b_{3}\left(\frac{D_{t}}{R_{t}+D_{t}} \log D_{t}+\right.$

$+\frac{R_{t}}{R_{t}+D_{t}} \log R_{t-1}-\log d_{t}-b_{1} \log P I_{t}$

El objetivo del autor es demostrar empiricamente que el coelicienle $b_{3}$ es estadísticamenle distinto de cero en ambas equaciones. La ecuación 9 la estimó direclamenle y a la ecuación 10 le aplió un procedimiento iteralivo asignándole valores sucesivos $a b_{1}$ hasta encontrar aquel valor que maximiza la capacidad explicaliva del modelo. El resultado de las estimaciones en el perlodo 1960-1975 fue el siguienle:

$\left(R_{t} / R_{t}+D_{t}\right)\left(\Delta \log R_{t}-\Delta \log R_{t-1}\right)=-0.4\left(D_{t} / R_{t}+D_{t}\right) \Delta \log D_{t}+$

$\left.\left(R_{t} / R_{t}+D_{t}\right) \Delta \log R_{t-1} \cdot \Delta \log d^{d}\right)+1.0 \Delta \log P_{t}-1.5 \Delta \log P I_{t-1}$

$1.2 \Delta \log P_{t-1}$

$\mathrm{R}^{2}=0.5$

$D-W=2.2$

$\log P_{t}-1.1 \log P_{t}=1.5\left(1.1 \log P_{t-1}-\log P_{t-1}\right)+0.6\left(\frac{D_{t}}{A_{t}+D_{t}} \log D_{t}+\right.$

$\left.+\frac{R_{t}}{A_{t}+D_{t}} \log A_{t-1}-\log m_{t}^{d}-1.1 \log P I_{t}\right)$ 
$\mathrm{R}^{2}=0.61$

$D-W=2.22$

El autor concluye que el modelo propuesto liene satisfactoria capacidad explicativa y que ambas ecuaciones muestran que el coeficiente $b_{3}$, a través del cual la oferta monelaria transmite su impulso a los precios, posee significación estadistica, es decir, es estadislicamente diferente de cero y aproximadamente igual a 0.60 . En consecuencia, un exceso de oferta monetaria provoca un aumento en el nivel de precios por encima del nivel de precios exógenos según la siguienle expresión:

$$
P_{t}=P^{*}+0.60 E O_{t}
$$

En lo que sigue aplicaré los tres modelos anteriores a la economía salvadorefía.

En estas aplicaciones se han respetado las variables y las luentes que se utilizaron en las versiones originales que se presentaron en la primera parte de esta sección.

1. El modelo de Harberger. (1973-1986)

El resultado empírico de este modelo fue el siguienle:

$\dot{\mathrm{P}}_{\mathrm{t}}=6.23-0.69 \hat{\mathrm{Y}}_{\mathrm{t}}-0.07 \hat{\mathrm{M}}_{\mathrm{t}}+0.63 \dot{\mathrm{M}}_{\mathrm{t}-1}+0.72 \hat{\mathrm{P}}_{\mathrm{t}}-0.06 \hat{\mathrm{W}}_{\mathrm{t}}$

$\begin{array}{lllll}(1.2) & (-1.6) \quad(-0.2) \quad(1.8) \quad(2.0) \quad(-0.3)\end{array}$

$\mathrm{A}^{2}=0.49$

$\overline{\mathrm{P}}^{2}=0.17$

$D-W=1.62$

La capacidad explicativa del modelo es baslante pobre, $\mathrm{R}^{2}=0.49$. Excepluando la aceleración de la ińlación que resultó ser estadísticamente distinta de cero al nivel del $10 \%$, lodos los demás coeficientes no fueron estadisticamente distintos de cero. Además, la oferta de dinero corriente no tiene ni la magnitud ni el signo teóricamente correctos; leóricamente cabría esperar signo posilivo y un valor cercano a la unidad. Por el contrario, la olerla rezagada un período sí liene el signo y la magnitud relativamenle correctos aunque no es estadisticamente significativo, en todo caso no es plausible que la olerla de dinero comience a afectar los precios un afio después. 
La presencia signilicativa de la aceleración de la inflación más que representar el papel del coslo de tener dinero como la leoria argumentaria, me parece que se debe a un efecto auto regresivo, es decir a la participación de la inflación rezagada como explicación de la misma inflación.

\section{El modelo de Nugent y Glezakos (1975-1986)}

La estimación de este modelo requiere el cálculo previo de la inflación esperada y del ingreso permanente. La primera variable se calculo en la sección anterior; la segunda se ha calculado aplicando el mismo modelo de expectalivas adaptativas por medio de la minimización de los errores de pronóstico que se utilizó en el cálculo de la inflación esperada. Ambas series se reportan en el apéndice. Los resultados de la estimación se muestran a continuación:

$$
\begin{aligned}
& \dot{\mathrm{P}}_{\mathrm{t}}=0.29 \hat{\mathrm{M}}_{\mathrm{t}}+0.27 \dot{\mathrm{M}}_{\mathrm{t}-1}+2.11 \hat{\mathrm{Y}}_{\mathrm{t}}+0.40 \dot{\mathrm{E}}_{\mathrm{t}} \\
& \mathrm{R}^{2}=0.69 \\
& \mathrm{D}-\mathrm{W}=2.1) \quad(4.7) \quad(3.6) \quad(3.7) \\
& \dot{\mathrm{P}}_{\mathrm{t}}=-0.12 \hat{\mathrm{M}}_{\mathrm{t}}-0.08 \hat{\mathrm{M}}_{\mathrm{t}-1}+0.30 \hat{\mathrm{P}}_{\mathrm{t}}-0.29 \hat{\hat{P}}_{\mathrm{t}} \\
& \quad(-4.2) \quad(-2.8) \\
& \mathrm{R}^{2}=0.76 \\
& \mathrm{D}-\mathrm{W}=2.90
\end{aligned}
$$

Las ecuaciones ajustan bastante bien y todos los coeficientes son esladísticamente significativos. Las ofertas de dinero corriente y rezagadas presentan los signos adecuados en la ecuación de precios, aunque no en la ecuación del ingreso transitorio. Ciertamente, en teoría se espera que aumentos en la cantidad de dinero provoquen en el corlo plazo aumentos de precios y de ingreso, la segunda ecuación estimada muestra lo contrario.

El hecho que el modelo incorpore la simultaneidad en la determinación de los precios y del ingreso mejora los resultados estimados acerca del papel del dinero en el proceso inflacionario. Sin embargo los resultados aún son pobres; ésto se puede apreciar en la lorma reducida 
de la ecuación de precios. Esta se obliene sustiluyendo la ecuación del ingreso en la ecuación de precios.

$$
\dot{\mathrm{P}}_{\mathrm{t}}=0.04 \dot{\mathrm{M}}_{\mathrm{t}}+0.10 \dot{\mathrm{M}}_{\mathrm{t}-1}+0.40 \dot{\mathrm{E}}_{\mathrm{t}}+0.63 \dot{\mathrm{P}}_{\mathrm{t}}-0.62 \dot{\mathrm{P}}_{\mathrm{t}}
$$

La forma reducida nos muestra que el impacto monetario en el corto plazo es bastante pobre. Un aumento de $1 \%$ en la oferta de dinero sólo aumenta los precios en el mismo periodo en $0.04 \%$ y en el siguiente en $0.10 \%$. Esto se explica según los resultados empíricos porque el aumento del dinero tiene eleclos sobre la producción en el corto plazo, los cuales reducen el impacto sobre los precios.

Los resultados empíicos, pues, no apoyan la afirmación tradicional de que la inflación en El Salvador sea provocado por razones monetarias. Pasemos a aplicar a continuación el modelo del enfoque monetario de la balanza de pagos a la hisloria reciente de la economía salvadorefia.

\section{El enfoque monetario de la balanza de pagos (1975- 1986)}

La especilicación del modelo dilerirá ligeramente del mostrado en la primera parte de esta sección, debido a que en los últimos años las reservas internacionales han presentado valores negativos volviendo imposible la oblención de logaritmos para esta variable. Por esla razón el modelo se ha especificado sin logaritmos, lo cual considero que no afecta fundamentalmente los resultados obtenidos ni las conclusiones que de aquí se deriven.

Primeramente se estimó la demanda de dinero siguiendo la especificación de Luis de Sebastián. El resultado oblenido se muestra a continuación.

$$
\begin{aligned}
\log m^{d}= & -6.9+1.40 \log Y_{t}-\left.0.92 \log \right|_{t}+1.20 \log P_{t} \\
& (-7.0) \quad(9.0) \quad(-0.28) \\
R^{2}= & 0.996 \\
D-W= & 2.20
\end{aligned}
$$

donde: $I_{t}=$ tasa de interés

Con este cálculo previo, procedl a estimar la ecuación 10, utilizando el mismo mélodo iterativo del autor, dándole valores $a b_{1}$, partiendo de cero y en incrementos de 0.1. el mejor resultado oblenido fue el siguiente: 


$$
\Delta \mathrm{P}_{\mathrm{t}}-1.6 \Delta \mathrm{Pl}_{\mathrm{t}}=-1.18\left(1.6 \Delta \mathrm{Pl}_{\mathrm{t}-1}-\Delta \mathrm{P}_{\mathrm{l}-1}\right)+0.009\left(\Delta \mathrm{D}_{\mathrm{t}}+\Delta \mathrm{P}_{\mathrm{t}-1}\right.
$$

$$
\mathrm{A}^{2}=0.93
$$

$$
\left.-\Delta \pi^{d_{1}}-1.6 \Delta P I_{t}\right)
$$

$D-W=1.80$

Los resultados son relativamente satisfaclorios; la capacidad explicativa del modelo así lo indica. Los signos de los coeficientes del componente de ajusle hacia los precios internacionales son adecuados; el signo negativo del primer término asegura que cada vez que los precios domésticos difieran de los internacionales algún ajuste entrará en acción, aunque leóricamente deberia ser inferior a la unidad para garantizar la convergencia. El coeficiente $b_{1}$ (1.6) deberia haber estado más cercano a la unidad.

Lo más importante de la estimación es que $b_{3}$ ha resultado estadisticamente signilicativo al nivel del $10 \%$. Esto indica, como fue en el caso de Sebastián, que el exceso de olerta de dinero tiene incidencia en los precios inlernos; sin embargo, en dilerencia con el anterior estudio, la magnitud del coeficiente $b_{3}$ es considerablemenle pequefia (0.009). Si comparamos el resullado final oblenido por de Sebastián con nuestro resultado, podremos contrastar la diferencia signilicaliva en la importancia del exceso de oferta monetaria.

a. Resultado anterior (1960-1975)

$$
P_{t}=P_{-t}+0.60 E O_{t}
$$

b. Resultado actual (1975-1986)

$$
P_{t}=P_{\cdot t}+0.009 \mathrm{EO}_{\mathrm{t}}
$$

La dilerencia es evidente. Aunque el resultado aclual muestra que el exceso de olerta monetaria tiene influencia en los precios domésticos, la magnitud de su impacto ha sido considerablemente inierior a la que pudo lener en el período anlerior. Apoya favorablemente nuestro resultado el hecho que la inflación ha estado presente a lo largo de lodo el periodo analizado; no es éste el caso del estudio anterior, el cual cubrió únicamente 3 años de verdadera inflación. 
Hemos arribado a resultados similares a los que obluvimos con el modelo de Nugent y Glezakos. El exceso de creación de dinero tiene alguna influencia en el crecimiento de los precios, pero su impacto carece del vigor necesario para llevarnos a concluir que la inflación en El Salvador sea un fenómeno monetario.

\section{Consideraclones finales}

El presente trabajo nos ha permitido comprobar la robustez de un modelo de costos para explicar el reciente proceso inflacionario salvadoreho. Asimismo, hemos verificado las dificultades de los modelos monetaristas para realizar similar larea. Por un lado, el modelo monelarisla tradicional de economía cerrada se encuentra limitado en su propia especilicación, tanto por no considerar la simultaneidad en la determinación de los precios y el ingreso, como por no considerar el sector externo. Por otro, los modelos que sí incorporan estos elementos muestran impactos insuficientes de los excesos de oferta monetaria sobre los precios para estableer el carácter monetario de la inllación. Sobre la base de eslos resuliados podemos escoger el modelo de costos como el mejor para explicar el proceso inflacionario salvadoreno ${ }^{17}$.

El modelo de costos nos ha senalado que la inflación en El Salvador es un proceso resultante de la acción de lactores primarios, aquellos que no son ellos mismos productos de la inflación: el crecimiento de los precios de las importaciones, la caida en la productividad del trabajo y el crecimiento del sector consirucción; y de factores inducidos, los que a su vez son productos del proceso inflacionario y que Iransmiten al proceso cierto carácler inercial: las expectativas inflacionarias, la devaluación del tipo de cambio y los aumentos salariales.

Por la evidencia podemos alirmar que los factores que más influencia han tenido en el proceso inflacionario han sido los precios de las importaciones, en la década pasada, y la caida de la productividad del trabajo y las devaluaciones, en la actual.

Asimismo, la diversidad de factores inflacionarios nos da claridad sobre la complejidad del lenómeno. En un pais pequeno, subdesarrollado y oligopólico, la inflación es algó más que solamente un desajuste entre el crecimiento de la olerta de dinero y el crecimiento del producto, de igual manera lo son las medidas correctivas.

Un programa antiinflacionario debe comprender, entonces, medidas de corto, mediano y largo plazo. Entre las primeras, son necesarias algunas medidas que devuelvan certidumbre a los agentes económicos para combatir las expectativas inflacionarias; el reciente anuncio de que durante 1988 no habrá devaluación ni aumentos de impuestos contribuirá en esla dirección. También es necesario proceder a descentralizar 
el área de construcción habitacional e industrial. En el mediano plazo, se requiere un programa de inversiones en consonancia con las restricciones del sector externo que contribuya a aumentar la olerta doméstica, tanto agricola como manufacturera, y a recuperar la productividad de el trabajo. $Y$ en el largo plazo, un plan de inversiones que impulse una mayor articulación entre los sectores productivos y que disminuya la dependencia externa de las importaciones para reducir la vulnerabilidad en la transmisión de la inllación importada.

También es importante senalar que en el corto plazo pueden ejecutarse medidas que impidan la tendencia regresiva en la distribución del Ingreso que la inflación pone en marcha. El impacto inflacionario del aumento de salarios debe evaluarse contra el cosio político de su congelación. El modelo que aqul se ha validado muestra que aquel impacto es relalivamente pequeño $(0.25)$ y que en consecuencia una política de protección del ingreso por esle medio es económicamente legítima.

El gran deterioro experimentado recientemente por la economia ha puesto de manifieslo la urgencia de medidas de política económica que impulsen la recuperación de los niveles de actividad y tiendan a atenuar los grandes desequilibrios macroeconómicos. La elaboración de lales medidas requiere, como esta investigación lo ha demostrado, de avanzar en los análisis de las lormas concretas en que los tenómenos económicos se desarrollan en El Salvador, debiendo eslar dispuestos a romper con tórmulas pre-establecidas y a incorporar las condiciones institucionales y socioeconómicas especílicas de la realidad salvadorefía. Si esta investigación ha contribuido a tomar conciencia de esla necesidad, el esfuerzo habrá sido bien compensado.

\section{NOTAS}

- El autor agradece los comentarios de Luis René Cáceres, Luis de Sebastián, Nolvia Saca, William Pleitez y Carlos Briones a una versión preliminar de este trabajo; asimismo, la colaboración de Ralael Lemus, Carlos Moreno y Orlando Martinez en la construcción de algunas series y estimaciones econométricas.

1. De Sebestián (1986) abordó el tema desde el enfoque monetario de la balanza de pagos; Siri y Dominguez (1979) y MIPLAN (1987) desde una perspectiva estructuralista.

2. A nivel tórico la discusión se centra en tomo a si es la cantidad de dinero la que determina el nivel de precios o a la inverse. La primera posición está representada por Milon Friedman (1956) y la segunda por Nicholas Kaldor (1983).

3. En un estudio reciente se ha demostrado emplricamente que mientras que las elasticidades ingreso de la producción agrlcola de alimentos decayeron en la década del setenta respecto a las del sesenta, las elasticidades ingreso de las importaciones aumentaron. Lorty Trigueros (1987).

4. Una encuesta incustrial realizada por el Ministerio de Planificación en 1983 con una muestra de 1062 establecimientos manufactureros de todos los tamaños, mostró que 
tanto en 1982 como en 1983 el promedio de tumos diarios trabajados lue 1.47 sobre 3 y que el coefieiente de aprovechamiento de la capacidad instalada lue alrededor de $67 \%$. Si tomemos en cuenta que de 1978, año de mayor nivel de producción de la manulactura, a 1983, la producción manufacturera se contrajo en $29 \%$, que la mayor parte de esta contracción se debió al cierre completo de muchos establecimientos y que durante este perlodo no hubo nuevas inversiones, se puede inferir que ya en 1978, es decir, antes de la crisis, la menulactura salvadoreña operaba con capacidad ociose.

5. A resultados similares llegó una investigación de campo que realizó en la industria inglesa, entrevistando 38 empresarios, un equipo de la Universidad de Oxlord en 1939. Este estudio mostró que una gran proporción de las empresas no hace intentos por igualar el costo y el ingreso marginal; que generalmente hay una luerte tendencia entre los empresarios a fijar los precios directamente a un nivel que consideran como el de su costo total y que los precios esl fijados tienen iendencia a ser estables y a cambiarse si hay un cambio significativo en los salarios o en el costo de la materia prima, pero no en respuesta a moderados o temporales desplazamientos de la demanda. Hall y Hitch (1939).

6. La misma encuesta de MIPLAN mostró que más del $14 \%$ de todos los establecimientos reciblan en 1983 financiamiento privado nacional, es decir, no bancario, lo cual indica que algún tipo de financiamiento informal está presente. En todo caso esta caracteristica institucional requiere mayor investigación emplrica antes de sacar condusiones.

7. Kaldor (1967). Más discusión teórica y emplrica puede encontrarse sobre esta "ley" en Kaldor (1966), Rowthom (1979), Dixon y Thirwall (1975) y Verdoom (1980).

8. El papel que Smith (1982) y Marx (1980) asignaron a los rendimientos crecientes a escala dentro del análisis económico, desapareció con el surgimiento de los teóricos marginalistas debido a las limitaciones que impuso la estructura anallica. Estas dificultades se expusieron claramente en Marshall (1957). Más recientemente han sido expuestas en Arrow (1962).

9. Allin Young (1928) enlatizó el carácler macroeconómico de esta relación. Las economlas de escala se derivan no solemente de la expansión de una industria sino principaimente de une expansión industrial generalizada.

10. El importante rol de las expectativas en el comportamiento económico lue planteado por Marshall (1923), Keynes (1930), Hicks (1976) y Fisher (1930). Tanto Fisher como Hicks enfatizaron el papel determinante de los valores corrientes y pasados en la formación del valor futuro de las variables. Fue Cagan (1956) quien lundamentó y formalizó la hipotesis de las expectativas adaptativas como un proceso de aprendizaje por experiencia a partir de las ideas de Fisher y Hicks. Recientemente ha cobrado presencia la hipótesis de las expectativas racionales, según la cual los agentes económicos sobre la base de una intormación completa conocen racionalmente los electos huturos de la pollica econónica. Muth (1961) y Lucas (1972).

11. La aplicación de minimos cuadrados ordinarios a una ecuación en donde aparece la veriable dependiente con rezagos como variable explicativa y en donde hay perturbaciones serialmente correlacionadas produce estimadores sesgados e inconsistentes. Johnston (1979).

12. Nugent y Glezako6 (1979) aplican el método, seňalando que "es aceptado generalmente en teorla economica que un eriterio razonable que los individuos usan en pronosticar valores huturos en contexto de incertidumbre es minimizar las pérdidas esperadas de los errores de pronóstico"

13. El modelo de expectativas adaptativas puede reordenarse de la siguiente manera:

$$
\hat{P}_{\mathrm{t}}=\mathrm{B} \hat{\mathrm{P}}_{\mathrm{l}-1}+(1-\mathrm{B}) \dot{\mathrm{P}}_{\mathrm{t}-1}^{\theta}
$$


La condición para que esto modelo converja a $\dot{P}_{k 1}$ es que (1-B) < 1, Wallis (1970). Entonces la condición se puede transformar en $-1<|1-\theta|<1$, b cual nos deja con $0<$ $B<2$.

En consecuencia, si $0<B<1$, po converjira monotónicamente a $\dot{P}_{t-1}$ si $1<B<2$, converjira oscilatoriamente a $\hat{P}_{\mathrm{t}-1}$. Este último es nuestro caso.

14. Resultados simileres ha encontrado Van Wijnbergen (1982) en Corea del Sur.

15. Este tipo de función de olerta tue fomuledo y veriticado emplricamente por Lucas y Papping (1972) y aplicado por Lucas (1973). El fundemento de la función es que la oferta está constituida por un componente secular, el cual refleja la acumulación de capital y los cambios en la población, y por un componente clclico, et cual es el resultado de que le demanda se distribuye inequitativamente sobre los mercados, conduciendo a movimientos en los precios tanto relativos como generales; de esta manera, el componente varia con los precios relativos percibidos por los olerentes.

16. Estrictamente la expresión debe ser:

$\Delta \log h+\frac{A}{R+D} \Delta \log A+\frac{D}{R+D} \Delta \log D=\Delta \log P+\Delta \log m_{i}^{d}$

si $\Delta$ ogh $=0$, entonces:

$$
\frac{R}{R+D} \Delta \log R+\frac{D}{R+D} \Delta \log D=\Delta \log P+\Delta \log m_{i}^{d}
$$

de donde:

$$
\frac{D^{*}}{R+D^{*}} \Delta \log _{t} D^{*}=\Delta \log P_{t}^{*}-\frac{A}{R+D^{*}} \Delta \log R_{t-1}+\Delta \log m_{t}^{d}
$$

17. -...una teorla es buena si pasa las pruebes emplricas mejor que alguna otra teorla, y mala si falla en hacerto asi. Si se desea aprender sobre teorla económica en relerencia a la evidencia emplrica, será necesario no una sino varias hipótesis que puedan ser puestas a pruebas simultáneamente, porque es la única forma en que las ideas teóricas útiles pueden seleccionarse de aquellas equivacadas" (Laidler, 1977, pág. 50). 


\section{APENDICE - A \\ DERIVACION OE LA TASA DE CAECIMIENTO DE LOS PRECIOS DE LA MANUFACTURA}

Sea:

$c$ = costo unitario.

w = salario por unidad de tiempo.

$\pi=$ productividad del trabajo.

$i$ = ase de interés nominal.

f = racción del costb unitario que se financia con credito bancario (s 1 ).

$q_{m}=$ cooficiente de insumos importados.

$q^{g}=$ coeficiente de insumos domésticos agrlcolas.

$\mathbf{q}^{m}=$ coeficiente de insumos domésticos manulacturados.

$P_{m}=$ precio de los insumos importados en moneda extranjera.

$E=$ Tipo de cambio nominal.

$p^{Q}=$ precio de los insumos domésticos agrícoles.

$p^{m}=$ precio de los insumos domésticos menufacturados.

a) La Ecusecín de Costos de la Menufacture.

Haciendo abstracción de la depreciación y de los impuestos, el costo unitario de la empresa será:

c = salario unitario + intereses unitarios + costo unitario de los insumos importados + costo unitario insumos manulacturados domésticos $+\infty$ costo unitario de los insumos agrloolas domésticos.

$$
\begin{array}{r}
c=\frac{w}{\pi}+i(f \cdot c)+q_{m} P_{m} E+q^{m} p^{m}+q^{2} p^{d} \\
q(1-i)=\frac{w}{\pi}+q_{m} P_{m} E+q^{m} p^{m}+q^{d} p^{d} \\
c=\frac{1}{(1-i n)}\left[\frac{w}{\pi}+q_{m} P_{m} E+q^{m} p^{m}+q^{q} p^{a}\right]
\end{array}
$$

Tomando diferencial.

$$
d c=\left[\frac{w}{\pi}+q_{m} P_{m} E+q^{m} p^{m}+q^{a} p^{a}\right] \cdot d\left(\frac{1}{(1-i)}\right)+\frac{1}{(1-i)} d\left[\frac{w}{\pi} q_{m} P_{m} E+q^{m} p^{m}+q^{a} p^{a}\right]
$$

Llamemos:

$$
A=\frac{w}{\pi}+q_{m} P_{m} E+q^{m} p^{m}+q^{q} p^{a}
$$

Entonces:

$$
d c=A d\left(\frac{1}{(1-i f)}\right)+\frac{1}{(1-i f)} d\left(\frac{w}{\pi}+q_{m} P_{m} E+q^{m} p^{m}+q^{d} p^{a}\right)
$$




$$
\begin{aligned}
& d c=A \frac{-(-1 d)}{(1-i)^{2}}+\frac{1}{1-i f} d\left(\frac{w}{\pi}+q_{m} d\left(P_{m} E\right)+q^{m} d p^{m}+q^{d} p^{a}\right) \\
& d c \frac{(1-i f)}{A}=\frac{t d}{(1-i i)}+\frac{1}{A}\left[d\left(\frac{w}{\pi}\right)+q_{m} d\left(P_{m} E\right)+q^{m} d p^{m}+q^{2} d p^{d}\right]
\end{aligned}
$$

De la ecuación 2 se sabe que:

$$
c=\frac{A}{1-i f}
$$

Entonces (3) se convierte en:

$$
\begin{aligned}
& \frac{d c}{c}=\frac{t d i}{(1-i f)}+\frac{1}{A}\left[d\left(\frac{w}{\pi}\right)+q_{m} d\left(p_{m} E\right)+q^{m} d p^{m}+q^{2} d p^{a}\right] \quad \text { (4) } \\
& \frac{d c}{c}=\frac{1}{1-i f} \cdot d i+\frac{1}{A} \cdot q^{m} \cdot d^{m}+\frac{1}{A}\left[d\left(\frac{w}{\pi}\right)+q_{m} d^{(}\left(P_{m} E\right)+q^{2} d p^{a}\right]
\end{aligned}
$$

Como se ve en 5 el precio de la industria se retroalimenta a los costos; su influencia dependerá del coeficiente insumo producto correspondiente (podrla determinarse de la matriz insumo producto dol Banco Central).

La hipótesis microeconómica es que los precios se fijan agregando un margen de ganancia a los costos unitarios.

Es decir:

$p^{m}=$ costo unitario + margen de ganancia .

$p^{m}=c . a$

donde: $\mathbf{a}=$ margen de ganancia $($ mark-up) $>1$.

Entonces:

$\frac{d p^{m}}{p^{m}}=\frac{d c}{c}+\frac{d g}{a}$

Sustituyendo en (5):

$\frac{d c}{c}=\frac{t}{1-f} \cdot d i+\frac{1}{A} \cdot q^{m} p^{m}\left(\frac{d c}{c}+\frac{d a}{a}\right)+\frac{1}{A}\left[d\left(\frac{w}{\pi}\right)+q_{m} d\left(p_{m} E\right)+q^{a} d p^{a}\right]$

Ordenando:

$\frac{d c}{c}\left(1-\frac{1}{A} q^{m} p^{m}\right)=\frac{1}{1-i l} d i+\frac{1}{A}\left[d\left(\frac{w}{\pi}\right)+q_{m} d\left(p_{m} E\right)+q^{a} d p^{a}+q^{m} p^{m} \frac{d a}{a}\right]$

llamando:

$B=1-\frac{1}{A} q^{m} p^{m}$ 
Y sustityyendo:

$$
\frac{d c}{c}=\frac{1}{B} \frac{1}{1-i f} d+\frac{1}{A B}\left[d\left(\frac{w}{\pi}\right)+q_{m} d\left(p_{m} E\right)+q^{2} d p^{a}+q^{m} p^{m} \frac{d a}{a}\right]
$$

Multiplicando y dividiendo cada término de la derecha por la veriable apropiada, se obtiene:

$$
\begin{aligned}
& \frac{d c}{c}=\frac{1}{B} \frac{\text { if }}{1-i f} \frac{d i}{i}+\frac{(w / \pi) d(w / \pi)}{A B(w / \pi)}+\frac{q m P m E}{A B} \frac{d(p m E)}{(p m E)}+\frac{q^{A} p^{B}}{A B} \frac{d p^{A}}{p^{a}}+\frac{q^{m} p^{m}}{A B} d a \\
& \hat{c}=\frac{\text { if }}{B(1-i f)} i+\frac{(w / \pi)}{A B}(w / \pi)+\frac{q_{m} p_{m} E}{A B}(p m E)+\frac{q^{a} p^{a}\left(p^{a}\right)}{A B} \frac{q^{m} p^{m}(a)}{\overline{A B}}
\end{aligned}
$$

donde ^ sobre una variable indica su tasa de cambio. desarrollemos el producto AB.

$$
A B=A\left(1-\frac{1}{A} q^{m} p^{m}\right)=A-q^{m} p^{m}
$$

pero: $\quad A=w / \pi+q_{m} p_{m} E+q^{m} p^{m}+q^{d} p^{q}$

Sustituyendo y simplificando:

$$
A B=W / \pi+q_{m} P_{m} E+q^{2} p^{2}
$$

Desarrollemos también:

$$
\frac{f}{B(1-i f)}=\frac{f i}{\left(1-\frac{1}{A} q^{m} p^{m}\right)(1-i f)}=\frac{A f}{\left(A-q^{m} p^{m}\right)(1-i f)}
$$

Sabemos de 2 que:

$$
c=\frac{A}{1-i I}
$$

Sustibyendo:

$$
\frac{f i}{B(1-i f)}=\frac{c f i}{\left(w / \pi+q_{m} P_{m} E+q^{a} p^{a}\right)}
$$

Sustituyendo 7 y 8 en 6 :

$$
\begin{aligned}
& \dot{c}=\frac{c f i}{\left(w / \pi+q_{m} P_{m} E+q^{a} p^{a}\right)} i+\frac{w / \pi}{\left(w / \pi+q_{m} P_{m} E+q^{a} p^{a}\right)}(w / \pi)+ \\
& \frac{q_{m} P_{m} E}{w / \pi+q_{m} P_{m} E+q^{a} p^{a}}(P m E)+{\frac{q^{a} p^{a}}{w / \pi+q_{m} P_{m} E+q^{a} p^{a}}}^{\dot{p}_{a}}+{\frac{q^{m} p^{m}}{w / \pi+q_{m} P_{m} E+q^{a} p^{a}}}_{(a)}^{(a)}
\end{aligned}
$$


Llamemos $c^{*}=w / \pi+q_{m} P_{m} E+q^{a} p^{a}$

que representa el costo unitario excluyendo el costo del crédito y de los insumos manufactureros.

$$
\dot{c}=\frac{c \hat{n}}{c^{*}} \hat{i}+\frac{w / \pi}{c^{*}}(w / \pi)+\frac{q m P_{m} E}{c^{*}} \quad(P \hat{m} E)+\frac{q^{a} p^{a}}{c^{*}}\left(\dot{p}^{a}\right)+\frac{q^{m} p^{m}}{c^{*}}(\hat{a})
$$

La ecuación (9) indica que:

$$
\hat{c}=t\left(\hat{i}, w \hat{\pi}, p_{m} \dot{m} E, \dot{p} \mathbf{a}, \hat{a}\right)
$$

La tasa de variación de los costos depende de las variaciones de las variables indicadas, cada una ponderada por el peso que bene sobre el costo c*.

b) La Ecuación de Preclos de la Manufactura.

Sabemos que $p^{m}=c a$

de donde: $\dot{p}^{m}=\dot{c}+\hat{\mathbf{a}}$

Sustituyendo 9 en 10

$$
\dot{p}^{m}=\frac{c f i}{c^{*}} \hat{\imath}+\frac{w / \pi}{c^{*}}(w / \pi)+\frac{q_{m} P_{m} E}{c^{*}}\left(p_{m} E\right)+\frac{q^{a} p^{a}}{c^{*}}\left(\dot{p^{\theta}}\right)+\left(\frac{q^{m} p^{m}+1}{c^{*}}\right)(\hat{a})
$$

Que se puede expresar:

$$
\hat{p}^{m}=a_{i} \hat{\imath}+a_{w}(w / \pi)+a_{m}(P \dot{m E})+a_{p a} \dot{P}^{a}+a_{a} a ́ a
$$


APENDICE B

SERIES UTIUZADAS

\begin{tabular}{|c|c|c|c|c|c|c|c|c|c|c|c|c|c|c|c|c|}
\hline & 1971 & 1972 & 1973 & 1974 & 1975 & 1976 & 1977 & 1978 & 1979 & 1990 & 1991 & 1982 & 1983 & 1984 & 1985 & 1986 \\
\hline $\begin{array}{l}\text { Intia da } \\
\text { Pracios } \\
\text { (1) }\end{array}$ & 47.53 & 48.29 & 5̣1.37 & 60.05 & $71.5 i$ & 76.58 & 85.63 & 96.97 & 108.69 & 127.5 & 146.44 & 163.59 & 185.07 & 206.74 & 252.86 & 333.64 \\
\hline $\begin{array}{l}\text { Salaio minti- } \\
\text { mo promo- } \\
\text { dado } \\
\text { (C) }\end{array}$ & 3.20 & 3.20 & 3.65 & 4.54 & 5.15 & 5.65 & 6.27 & 7.00 & 7.63 & 10.33 & 11.00 & 11.00 & 11.00 & 12.33 & 13.00 & 15.00 \\
\hline $\begin{array}{l}\text { Tera de } \\
\text { inerts } \\
\text { (1) }\end{array}$ & 10.0 & 10.0 & 10.0 & 11.0 & 11.0 & 11.0 & 11.0 & 13.0 & 13.0 & 13.0 & 13.0 & 13.0 & 13.0 & 13.0 & 13.0 & 15.0 \\
\hline $\begin{array}{l}\text { Tpo da cambio } \\
\text { (2) }\end{array}$ & 2.5 & 2.5 & 25 & 2.5 & 2.5 & 25 & 2.5 & 2.5 & 2.5 & 2.5 & 2.5 & 2.5 & 2.85 & 2.92 & 3.58 & 5.00 \\
\hline $\begin{array}{l}\text { Olarta da difo } \\
\text { o }(\mathrm{N} / \mathrm{C}) \\
\text { (1) }\end{array}$ & 686.80 & $82 B .10$ & 984.40 & 1150.69 & 1306.52 & 1850.31 & 2110.12 & 2311.44 & 2550.96 & 2692.40 & 2960.60 & 3249.60 & 3578.90 & 4281.80 & 5491.10 & 7037.40 \\
\hline
\end{tabular}

\& 
SERIES UTIUZADAS

\begin{tabular}{|c|c|c|c|c|c|c|c|c|c|c|c|c|c|c|c|c|}
\hline & 1971 & 1972 & 1973 & 1974 & 1975 & $19 \pi$ & 1977 & 1978 & 1979 & 1980 & 1981 & 1982 & 1980 & 1984 & 1985 & 1986 \\
\hline $\begin{array}{l}\text { Tesed de } \\
\text { creciniento } \\
\text { del sector } \\
\text { corsinucosion } \\
\text { (1) }\end{array}$ & -1.1 & 0.0 & .14 .8 & 5.9 & 49.3 & -9.4 & 35.6 & 6.4 & .2 .1 & -22.6 & .15 .3 & 42 & 2.0 & .5 .7 & 4.6 & 4.4 \\
\hline $\begin{array}{l}\text { Tasa de } \\
\text { cesinionto } \\
\text { del PB. } \\
\text { (1) }\end{array}$ & 4.8 & 5.5 & 5.1 & 6.4 & 5.5 & 3.9 & 6.1 & 6.3 & -1.7 & 8.7 & 8.3 & -5.7 & 0.8 & 1.5 & 2.8 & 10 \\
\hline $\begin{array}{l}\text { PB (1) } \\
\text { a precios } \\
\text { corsistartes } \\
\text { de } 1962\end{array}$ & 2508.8 & 2645.9 & 2779.0 & 2958.4 & 3122.9 & $\$ 246.9$ & 344.9 & 3664.7 & 3601.6 & 3289.3 & 3016.8 & 2847.7 & 2870.4 & 2912.9 & 2959.5 & 2989.1 \\
\hline $\begin{array}{l}\text { Iflerion } \\
\text { ospencin } \\
\text { (4) }\end{array}$ & 0.0 & 0.0 & 1.9 & 7.1 & 18.6 & 19.2 & 5.2 & 13.0 & 13.4 & 11.9 & 18.3 & 14.3 & 11.4 & 13.5 & 11.5 & 241 \\
\hline $\begin{array}{l}\text { inforde } \\
\text { precing do } \\
\text { alinerios } \\
\text { (1) }\end{array}$ & - & - & 52.2 & 61.2 & 73.6 & 76.9 & 85.7 & 94.9 & 100.9 & 130.3 & 159.3 & 169.6 & 192.3 & 219.5 & 260.9 & 344.0 \\
\hline
\end{tabular}


SERIES UTLIZADAS

\begin{tabular}{|c|c|c|c|c|c|c|c|c|c|c|c|c|c|c|c|c|}
\hline & 1971 & 1972 & 1973 & 1974 & 1975 & 1976 & 1977 & 1978 & 1979 & 1980 & 1981 & 1982 & 1983 & 1984 & 1985 & 1986 \\
\hline $\begin{array}{l}\text { Indloo de } \\
\text { precios vesidio } \\
\text { (1) }\end{array}$ & - & - & 51.2 & 55.9 & 72.1 & 80.5 & 87.7 & 85.5 & 108.7 & 124.1 & 150.3 & 175.1 & 197.9 & 216.9 & 270.2 & 3642 \\
\hline $\begin{array}{l}\text { Indice de } \\
\text { preclos } \\
\text { vivienda } \\
\text { (1) }\end{array}$ & - & - & 41.1 & 49.8 & 58.0 & 69.0 & 72.7 & 892 & 100.4 & 127.2 & 139.0 & 155.3 & 178.8 & 195.4 & 259.9 & 338.4 \\
\hline $\begin{array}{l}\text { Indice de } \\
\text { precios } \\
\text { miscoláneos } \\
\text { (1) }\end{array}$ & - & - & 50.5 & 59.6 & 652 & 67.1 & 82.0 & 94.8 & 100.3 & 122.1 & 135.4 & 152.5 & 167.8 & 181.9 & 214.1 & $284 . \theta$ \\
\hline $\begin{array}{l}\text { Componente } \\
\text { domestico de la } \\
\text { olerte de dinero } \\
\text { (2) }\end{array}$ & - & 299.6 & 390.0 & 610.0 & 6555 & 680.4 & 605.8 & $\pi 7.6$ & 1360.5 & 2329.6 & 2760.4 & 3120.3 & 2984.1 & 3240.0 & 3669.0 & 46320 \\
\hline $\begin{array}{l}\text { Peservas } \\
\text { internecionales } \\
\text { (2) }\end{array}$ & - & 112.0 & 106.7 & -11.8 & 56.6 & 236.3 & 392.5 & 3093 & -39.6 & -901.0 & -1332.2 & -1403.7 & -1326.8 & -1279.0 & -1180.0 & $\mid-1585.0$ \\
\hline
\end{tabular}


SEAIES UTILADAS

\begin{tabular}{|c|c|c|c|c|c|c|c|c|c|c|c|c|c|c|c|c|}
\hline & 1971 & 1972 & 1973 & 1974 & 1975 & 1976 & 1977 & 1970 & 1979 & 1960 & 1891 & 1902 & 1920 & 1994 & 1925 & 1906 \\
\hline $\begin{array}{l}\text { Exososo } \\
\text { de olerta } \\
\text { de dineroro } \\
\text { (4) }\end{array}$ & - & - & - & 207.2 & 602 & 86.5 & 93.6 & $\mathbf{3 1 0 . 5}$ & 509.9 & $\$ 1.3$ & $\$ 14.7$ & $\cdot 71.5$ & -2120 & 323.5 & 467.3 & 1057.4 \\
\hline $\begin{array}{l}\text { Producto } \\
\text { menutacturen } \\
\text { a precias } \\
\text { constentas } \\
1962 \text { (1) }\end{array}$ & 468.9 & 466.9 & 521.8 & 552.2 & 578.0 & $\mathfrak{2 8 . 6}$ & 661.5 & $\omega 1.5$ & 656.8 & 566.2 & 525.0 & 480.9 & 490.5 & 496.9 & 515.4 & 527.6 \\
\hline $\begin{array}{l}\text { Producto } \\
\text { manufacty- } \\
\text { reno tenden- } \\
\text { cial a p.c. } \\
1962 \text { (4) }\end{array}$ & 350.9 & 393.4 & $403 . \theta$ & 425.4 & 440.1 & 4720 & 497.2 & $\mathbf{5 2 0 . 7}$ & 551.7 & 567.1 & 6122 & 644.8 & 679.3 & 715.5 & 739.7 & 793.9 \\
\hline $\begin{array}{l}\text { Crecimiento } \\
\text { de la produc- } \\
\text { ividad del } \\
\text { trabajo. } \\
\text { (Por cient) } \\
\text { (4) }\end{array}$ & 4.70 & 1.22 & 4.91 & 3.42 & 2.16 & 6.66 & 2.77 & 1.99 & -8.47 & -14.70 & -14.36 & -1215 & 0.79 & -1.56 & 1.10 & 0.39 \\
\hline $\begin{array}{l}\text { Variación } \\
\text { del costb } \\
\text { salanial } \\
\text { unitario (4) }\end{array}$ & -4.70 & -1.22 & 9.15 & 20.96 & 11.27 & 6.94 & 4.41 & 9.65 & 20.33 & $46 . \circledast$ & 20.85 & 1215 & 0.79 & 13.65 & 4.30 & 15.77 \\
\hline
\end{tabular}


SEAIES UTIUZADAS

\begin{tabular}{|c|c|c|c|c|c|c|c|c|c|c|c|c|c|c|c|c|}
\hline & 1971 & 1972 & 1973 & 1974 & 1975 & 1976 & 197 & 1978 & 1979 & 1980 & 1981 & 1992 & 1980 & 1984 & 1985 & 1986 \\
\hline $\begin{array}{l}\text { holop de procices } \\
\text { USA } \\
\text { (2) }\end{array}$ & 49.1 & 50.8 & 50.9 & 59.8 & 65.3 & 6.1 & 73.6 & 79.2 & 86.1 & 100.0 & 110.4 & 117.1 & 120.9 & 16.1 & 130.5 & 134.2 \\
\hline $\begin{array}{l}\text { hodico de precios } \\
\text { Alemenis } \\
\text { (2) }\end{array}$ & 64.1 & 67.7 & 724 & $\pi .4$ & 820 & 85.6 & 69.7 & 91.1 & 94.9 & 100.0 & 106.3 & 111.9 & 115.6 & 118.4 & 121.0 & 120.1 \\
\hline $\begin{array}{l}\text { hocos de procies } \\
\text { (2) }\end{array}$ & 4.9 & 469 & 524 & 65.2 & 729 & 79.7 & 6.1 & 89.4 & 926 & 100.0 & 104.9 & 107.7 & 109.6 & 1121 & 114.4 & 115.5 \\
\hline $\begin{array}{l}\text { Indice de precios } \\
\text { Gualemeate } \\
\text { (2) }\end{array}$ & 3.9 & 40.1 & 45.6 & 53.2 & 62 & 66.6 & 74.8 & 81.0 & 90.2 & 100.0 & 111.4 & 111.8 & 116.8 & 120.8 & 143.4 & 210.2 \\
\hline $\begin{array}{l}\text { hdice de precios } \\
\text { Costa Pica } \\
\text { (2) }\end{array}$ & 36.9 & 39.6 & 44.4 & 57.8 & 67.0 & 70.2 & 73.1 & 77.5 & B4.7 & 100.0 & 137.1 & 260.6 & 345.6 & 366.9 & 445.1 & 518.7 \\
\hline
\end{tabular}




\begin{tabular}{|c|c|c|c|c|c|c|c|c|c|c|c|c|c|c|c|c|}
\hline & 1971 & 1972 & 1973 & 1974 & 1975 & 1976 & 1977 & 1978 & 1979 & 1960 & 1981 & 1982 & 1980 & 1984 & 1985 & 1996 \\
\hline $\begin{array}{l}\text { Importaciones } \\
\text { USA } \\
\text { (2) }\end{array}$ & 175.0 & 190.2 & 2725 & 433.6 & 470.3 & 5123 & (61.3 & 7927 & 717.9 & 483.7 & 624.1 & 561.9 & 706.1 & 611.3 & 813.4 & 1761.1 \\
\hline $\begin{array}{l}\text { Importaciones } \\
\text { Alemaria } \\
\text { (2) }\end{array}$ & 51.0 & 53.9 & 71.6 & 99.0 & 69.4 & 104.9 & 135.7 & 133.8 & 117.7 & 61.0 & 92.0 & 99.5 & S28 & 107.9 & 105.0 & 2122 \\
\hline $\begin{array}{l}\text { Importaciones } \\
\text { Japón } \\
\text { (2) }\end{array}$ & 73.2 & 76.6 & $\$ 0.7$ & 111.4 & 100.4 & 173.9 & $\mathbf{2 6 1 . 1}$ & 305.4 & 196.5 & 80.9 & 60.7 & 66.8 & 78.2 & 104.8 & 124.1 & 1665 \\
\hline $\begin{array}{l}\text { Importaciones } \\
\text { Guatemala } \\
\text { (2) }\end{array}$ & 104.8 & 119.2 & 149.4 & 175.6 & 210.9 & 264.9 & 320.0 & 375.1 & 451.7 & 634.0 & 618.7 & 524.8 & 428.1 & 468.7 & 374.1 & 543.3 \\
\hline $\begin{array}{l}\text { Importaciones } \\
\text { Cost Pica } \\
\text { (2) }\end{array}$ & 27.8 & 31.7 & 43.6 & 62.8 & 69.5 & 84.5 & 118.4 & 121.5 & 125.5 & 139.5 & 116.9 & 0.9 & 1072 & 116.7 & 135.3 & $182 \theta$ \\
\hline $\begin{array}{l}\text { hdice de precios } \\
\text { de principales } \\
\text { socios comercieles } \\
\text { (3) }\end{array}$ & 47.1 & 48.6 & 53.2 & 61.0 & 66.0 & 71.7 & 77.3 & 82.2 & 89.5 & 100.0 & 1123 & 128.7 & 135.7 & 1420 & 159.1 & 171.7 \\
\hline
\end{tabular}

FUENTES: 1. Revistas mensuales y memorias del Banco Central de Resona 2. Estadisticos Financieras internacionales. FMI.

3. Calaulado en bese a dabs oficiales.

4. Estimedos estadisticos del autor sobre la base de dabs oficiales. 


\section{REFERENCIAS}

Arrow, K.J. (1962). "The Economic Implications of Leaning by Doing". Review of Economic Studies.

Blejer, M. (1978). "The Short Run Dynamics of Prices and the Balance of Payments". American Economic Review, June.

Cagan, P. (1956). "The Monetary Dynamics of Hiperinflation" en "Studies in the Quantity of Money". Friedman, Milton. Chicago.

De Sebastién, L. (1986). "Inflación, Moneda y Balanza de Pagos on El Salvador. UCA Editores.

Dixon, R. and Thirtwall, A.P. (1975). "A model of Regional Growth Rate Diflerences in Kaldorian Lines". Oxford Economic Papers, July.

Fisher, I. (1930). "The Teory of Interest", New York.

Frenkel, J. and Johnson, H. (1976). "The Monetary Approach to the Balance of Payments". Alen Unwin, London.

Friedman, M. (1956). "The Quantity of Money -a Restatement en Studies in the Quantity Theory of Money. Univ. Chicago Press.

Godley, W.A.H. y Nordhaus, W.D. (1972). "Pricing in the Trade Cycle". Economic Journal, Sept

Hall, R.L. y Hitch, C.J. (1939). "Teorla de los Precios y Comportamiento de las Empresas". Oxford Economic Papers, $N^{0} 2$.

Harberger, A. (1963). "The Dynamics of Inflation in Chile" in "Measurement in Economics: Studies in Mathematical Economies and Econometrics in Memory of Yehuda Grunfeld", Stanlord.

Hicks, J. (1976). "Valor y Capital", F.C.E.

International Monetary Fund (1977). "The Monetary Approsch to the Balance of Payments". Washington, D.C.

Johnston, J. (1979). "Mótodos de Econometrla". Editorial Vicens-Vives, capitulo 10.

Kaldor, (1983). "Acerca del Monetarismo". Investigación Económica 166, OctubreDiciembre. México.

Kaldor, N. (1967). "The Rate of Increasing Returns in Industry" en "Strategic Factors in Economic Development, Comell University.

Kaldor, N. (1966). "Causes of the Slow Rate of Growth of the United Kingdom", Cambridge University Press.

Kalecki. M. (1982). "Ensayos Escogidos sobre la Dinámica de las Economlas Capitaistas", F.C.E.

Keynes, J.M. (1930). “A Treatise on Money", London.

Labini, S. (1966). "Oligopolio y Progreso Técnico", Oikos-Tau.

Ladman, J., Torrico, J. y Bunce, T. (1986). "Mercados Financieros Rurales de El Salvador. Movilización de Ahorros y Crédito Agropecuario*. Documento preparado para USAID/EI Salvador.

Laidler, D. (1977). "The Demand for Money-Theories and Evidence". Dun-Donnelley Publishing Corporation. New York.

Llor, M. y Trigueros, A. (1987). "La Olerta de Alimentos Agrícolas en El Salvador." Tesis de grado, UCA.

Lin, J. (1987). "The New Structuralist Critique of the Monetarist Theory of Inflation. The Case of the Philippines". Joumal of Development Economics 25.

Luces, R. (1972). "Expectation and the neutrattity of Money" Journal of Economic Theory.

Lucas, R. y Rapping. L. (1972). "Aeal Wages, Employment and the Price Level". Journal of Political Economy. Jan/Feb.

Lucas, A. (1973). "Some International Evidence on Output Inflation Trade Off". American Economic Review, 63, ND 3, June. 
Marshall, A. (1957). "Principios de Economfa". Ed. Aguilar.

Marshall, A. (1923). "Money, Credit and Commerce". London.

Marx, K. (1980). "El Capital." Siglo XXI.

MIPLAN, (1987). "Causas de la Inflación en El Salvador e Implicaciones Socio-Económices de una Nueva Devaluación del Colón". Mayo.

Muth, J. (1961). "Rationel Expectations and the Theory of Price Movernents". Econometrica, July.

Nugent, J. y Glezakos, C. (1979). "A Model of Inflation and Expectabives in Latin America". Joumal of Development Economies 6.

Pérez, M. y Contreras, L. (1987). "La Formación de Precios en la Manulactura Salvadorenta". Tesis de grado, UCA.

Rivera C.P. (1983). "La Concentración en la Manulactura Salvadoreña: Un Intento de Cuantificación". Tesis de grado, UCA.

Rowthom, R. (1979). 'A Note on Verdoom Law'. Economic Joumal, March.

Siri, G. y Dominguez, L. (1979). "Central American Accomodation to External Disruptions".

Smith, A. (1982). "Investigación sobre la Naturaleza y Causas de la Riqueza de las Naciones". F.C.E.

Spigelman, D. (1987). "Macroeconomic Instability of the Less Developed Couniry Economy when Bank Credit is Rationed". Intemational Finance Discussion Papers N0 305, March.

Steind, J. (1979). "Madurez y Estancamiento en el Capitalismo Norteamericano". Siglo XXI.

Taylor, L. (1983). "Structuralist Macroeconomics, Applicable Models for the Third World". Basic Books. Inc. New York.

Taylor, L. (1982). "IS/LM in the Tropics: Diagrammaties of the New Structuralist Macro Critique".

Van Wijnbergen, S. (1982). "Stagflationary Effects of Monetary Stabilization Policies. A Ouantitative Analysis of South Korea". Joumal of Development Economies 10. North Holland Publishing Company.

Vásquez, A. (1981). "Crecimiento Económico y Productividad en la Indusiria Manulacturera". Economla Mexicana CIDE.

Verdoom, P.J. (1949). "Fattori che regolano. Lo sviluppo della produltivita del Lavoro". L'Industria.

Verdoom, P.J. (1980). "Verdoorn's Law in Retrospecl: A Comment. June.

Wallis, K. (1979). "Introducción a le Econometrla". Alianza Universided.

Young, A. (1928). "Increasing Relurns and Economic Progress", Economic Journal, Docember. 